\title{
Sharp Convergence Rate of the Glimm Scheme for General Nonlinear Hyperbolic Systems
}

\author{
Fabio Ancona, Andrea Marson
}

March 2009

\begin{abstract}
Consider a general strictly hyperbolic, quasilinear system, in one space dimension

$$
u_{t}+A(u) u_{x}=0
$$

where $u \mapsto A(u), u \in \Omega \subset \mathbb{R}^{N}$, is a smooth matrix-valued map. Given an initial datum $u(0, \cdot)$ with small total variation, let $u(t, \cdot)$ be the corresponding (unique) vanishing viscosity solution of (1) obtained as limit of solutions to the viscous parabolic approximation $u_{t}+A(u) u_{x}=\mu u_{x x}$, as $\mu \rightarrow 0$. We prove the a-priori bound

$$
\left\|u^{\varepsilon}(T, \cdot)-u(T, \cdot)\right\|=o(1) \cdot \sqrt{\varepsilon}|\log \varepsilon|
$$

for an approximate solution $u^{\varepsilon}$ of (1) constructed by the Glimm scheme, with mesh size $\Delta x=\Delta t=\varepsilon$, and with a suitable choice of the sampling sequence. This result provides for general hyperbolic systems the same type of error estimates valid for Glimm approximate solutions of hyperbolic systems of conservation laws $u_{t}+F(u)_{x}=0$ satisfying the classical Lax or Liu assumptions on the eigenvalues $\lambda_{k}(u)$ and on the eigenvectors $r_{k}(u)$ of the Jacobian matrix $A(u)=D F(u)$.

The estimate (2) is obtained introducing a new wave interaction functional with a cubic term that controls the nonlinear coupling of waves of the same family and at the same time decreases at interactions by a quantity that is of the same order of the product of the wave strength times the change in the wave speeds. This is precisely the type of errors arising in a wave tracing analysis of the Glimm scheme, which is crucial to control in order to achieve an accurate estimate of the convergence rate as (2).
\end{abstract}




\section{Contents}

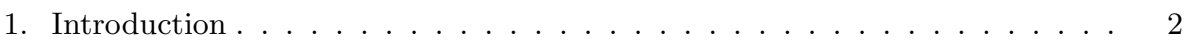

2. Preliminaries . . . . . . . . . . . . . . . . . 7

3. New wave interaction potential . . . . . . . . . . . . . . . . 13

4. Wave tracing for general quasilinear systems . . . . . . . . . . . . . . . 23

5. Conclusion . . . . . . . . . . . . . . . . . . . 28

\section{Introduction}

Consider a general strictly hyperbolic, $N \times N$ quasilinear system in one space dimension

$$
u_{t}+A(u) u_{x}=0,
$$

where $u \mapsto A(u)$ is a $\mathcal{C}^{2}$ matrix valued map defined from a domain $\Omega \subseteq \mathbb{R}^{N}$ into $\mathbf{M}^{N \times N}(\mathbb{R})$, and $A(u)$ has $N$ real distinct eigenvalues

$$
\lambda_{1}(u)<\cdots<\lambda_{N}(u) \quad \forall u .
$$

Denote with $r_{1}(u), \ldots, r_{N}(u)$ a corresponding basis of right eigenvectors. The fundamental paper of Bianchini and Bressan [9] shows that (1.1) generates a unique (up to the domain) Lipschitz continuous semigroup $\left\{S_{t}: t \geq 0\right\}$ of vanishing viscosity solutions with small total variation obtained as the (unique) limits of solutions to the (artificial) viscous parabolic approximation

$$
u_{t}+A(u) u_{x}=\mu u_{x x}
$$

when the viscosity coefficient $\mu \rightarrow 0$. In particular, in the conservative case where $A(u)$ is the Jacobian matrix of a flux function $F(u)$, every vanishing viscosity solution of (1.1) provides a weak solution (in a distributional sense) of

$$
u_{t}+F(u)_{x}=0
$$

satisfying an admissibility criterion proposed by T.P. Liu in [22,23], which generalizes the classical stability conditions introduced by Lax [20].

Definition 1. A shock discontinuity of the $k$-th family $\left(u^{L}, u^{R}\right)$, traveling with speed $\sigma_{k}\left[u^{L}, u^{R}\right]$, is Liu admissible if, for any state $u$ lying on the Hugoniot curve $S_{k}\left[u^{L}\right]$ between $u^{L}$ and $u^{R}$, the shock speed $\sigma_{k}\left[u^{L}, u\right]$ of the discontinuity $\left(u^{L}, u\right)$ satisfies

$$
\sigma_{k}\left[u^{L}, u\right] \geq \sigma_{k}\left[u^{L}, u^{R}\right] .
$$

Such a criterion needs to be imposed to rule out non-physical discontinuities, since weak solutions to Cauchy problems for (1.4) are not unique.

Given an initial datum with small total variation

$$
u(0, x)=\bar{u}(x),
$$


the existence of global weak admissible solutions to (1.4)-(1.6) was first established in the celebrated paper of Glimm [16] under the additional assumption that each characteristic field $r_{k}$ be either linearly degenerate (LD), so that

$$
\nabla \lambda_{k}(u) \cdot r_{k}(u)=0 \quad \forall u,
$$

or else genuinely nonlinear (GNL) i.e.

$$
\nabla \lambda_{k}(u) \cdot r_{k}(u) \neq 0 \quad \forall u .
$$

A random choice method, the Glimm scheme, was introduced in [16] to construct approximate solutions of the general Cauchy problem (1.4)-(1.6) by piecing together solutions of several Riemann problems, i.e. Cauchy problems whose initial data are piecewise constant with a single jump at the origin

$$
u(0, x)=\left\{\begin{array}{lll}
u^{L} & \text { if } & x<0 \\
u^{R} & \text { if } & x>0
\end{array}\right.
$$

Using a nonlinear functional introduced by Glimm, that measures the nonlinear coupling of waves in the solution, one can establish a-priori bounds on the total variation of a family of approximate solutions. These uniform estimates then yield the convergence of a sequence of approximate solutions to the weak admissible solution of (1.4)-(1.6). The existence theory for the Cauchy problem (1.4)-(1.6) based on a Glimm scheme was extended by Liu [24], Liu and Yang [25], and by Iguchy and LeFloch [19] to the case of systems with non genuinely nonlinear (NGNL) characteristic families that exhibit finitely many points of lack of genuine nonlinearity along each elementary curve, and by Bianchini [7] to general hyperbolic systems (1.1).

The aim of the present paper is to provide a sharp convergence rate for approximate solutions obtained by the Glimm scheme valid for general hyperbolic quasilinear systems (1.1), without any additional assumption on $A(u)$ besides the strict hyperbolicity (1.2). We recall that in the Glimm scheme, one works with a fix grid in the $t-x$ plane, with mesh sizes $\Delta t, \Delta x$. An approximate solution $u^{\varepsilon}$ of (1.4)-(1.6) is then constructed as follows. By possibly performing a linear change of coordinates in the $t-x$ plane, we may assume that the characteristic speeds $\lambda_{k}(u), 1 \leq k \leq N$, take values in the interval [0,1], for all $u \in \Omega$. Then, choose $\Delta t=\Delta x \doteq \varepsilon$, and let $\left\{\theta_{\ell}\right\}_{\ell \in \mathbb{N}} \subset[0,1]$ be an equidistributed sequence of numbers, which thus satisfies the condition

$$
\lim _{n \rightarrow \infty}\left|\lambda-\frac{1}{n} \sum_{\ell=0}^{n-1} \chi_{[0, \lambda]}\left(\theta_{\ell}\right)\right|=0 \quad \forall \lambda \in[0,1],
$$

where $\chi_{[0, \lambda]}$ denotes the characteristic function of the interval $[0, \lambda]$. On the initial strip $0 \leq t<\varepsilon, u^{\varepsilon}$ is defined as the exact solution of (1.4), with starting condition

$$
\left.u^{\varepsilon}(0, x)=\bar{u}\left(\left(j+\theta_{0}\right) \varepsilon\right) \quad \forall x \in\right] j \varepsilon,(j+1) \varepsilon[.
$$

The elementary waves of the corresponding Riemann problem do not interact within the the strip because the characteristic speeds $\lambda_{k}(u)$ take values in $[0,1]$. 
Next, assuming that $u^{\varepsilon}$ has been constructed for $t \in[0, i \varepsilon[$, on the strip $i \varepsilon \leq$ $t<(i+1) \varepsilon, u^{\varepsilon}$ is defined as the exact solution of (1.4), with starting condition

$$
\left.u^{\varepsilon}(i \varepsilon, x)=u^{\varepsilon}\left(i \varepsilon-,\left(j+\theta_{i}\right) \varepsilon\right) \quad \forall x \in\right] j \varepsilon,(j+1) \varepsilon[.
$$

Relying on uniform a-priori bounds on the total variation, we thus define inductively the approximate solution $u^{\varepsilon}(t, \cdot)$ for all $t \geq 0$.

One can repeat this construction with the same values $\theta_{i}$ for each time interval $[i \varepsilon,(i+1) \varepsilon[$, and letting the mesh size $\varepsilon$ tend to zero. Hence, we obtain a parametrized family of solutions $u^{\varepsilon}$ which converge, by compactness, to some limit function $u$ that results to be a vanishing viscosity solution of (1.1), (1.6) (cfr. [9]). In order to derive an accurate estimate of the convergence rate of the approximate solutions, it was introduced in [12] an equidistributed sequence $\left\{\theta_{\ell}\right\}_{\ell \in \mathbb{N}} \subset[0,1]$ enjoying the following property. For any given $0 \leq m<n$, define the discrepancy of the set $\left\{\theta_{m}, \ldots, \theta_{n-1}\right\}$ as

$$
D_{m, n} \doteq \sup _{\lambda \in[0,1]}\left|\lambda-\frac{1}{n-m} \sum_{m \leq \ell<n} \chi_{[0, \lambda]}\left(\theta_{\ell}\right)\right| .
$$

Then, there holds

$$
D_{m, n} \leq \mathcal{O}(1) \cdot \frac{1+\log (n-m)}{n-m} \quad \forall n>m \geq 1 .
$$

Here, and throughout the paper, $\mathcal{O}(1)$ denotes a uniformly bounded quantity, while we will use the Landau symbol $o(1)$ to indicate a quantity that approaches zero as $\varepsilon \rightarrow 0$. For systems (1.4) with GNL or LD characteristic fields, the $\mathbb{L}^{1}$ convergence rate of Glimm approximate solutions constructed with a sampling sequence enjoying the property (1.12) was shown in [12] to be $o(1) \cdot \sqrt{\varepsilon}|\ln \varepsilon|$. This error estimate was recently extended in $[5,17]$ to quasilinear systems $(1.1)$ satisfying the assumption

(H) For each $k \in\{1, \ldots, N\}$-th characteristic family, the linearly degenerate manifold

$$
\mathcal{M}_{k} \doteq\left\{u \in \Omega: \nabla \lambda_{k}(u) \cdot r_{k}(u)=0\right\}
$$

is either empty (GNL characteristic field), or it is the whole space (LD characteristic field), or it consists of a finite number of smooth, connected, hypersurfaces, and there holds

$$
\nabla\left(\nabla \lambda_{k} \cdot r_{k}\right)(u) \cdot r_{k}(u) \neq 0 \quad \forall u \in \mathcal{M}_{k}
$$

Notice that the Liu admissible solution of a Riemann problem for a system of conservation laws satisfying the assumption $(\mathrm{H})$ consists of centered rarefaction waves, compressive shocks or composed waves made of a finite number of Liu admissible contact-discontinuities adjacent to rarefaction waves. On the contrary, the solution of a Riemann problem for a general hyperbolic system (1.4) may well be a composed wave containing a countable number of rarefaction waves and Liu admissible contact-discontinuities. 
In the present paper we show that the same convergence rate valid for systems satisfying the assumption $(\mathrm{H})$ continue to hold even for Glimm approximate solutions of general quasilinear systems (1.1). Namely, our result is the following.

Theorem 1. Let $A$ be a $\mathcal{C}^{2}$ matrix valued map defined from a domain $\Omega \subset \mathbb{R}^{N}$ into $\mathbf{M}^{N \times N}(\mathbb{R})$, and assume that the matrices $A(u)$ are strictly hyperbolic. Then, for every compact set $K \subset \Omega$, there exists a constant $\delta_{0}>0$ such that the following holds. Given an initial datum $\bar{u} \in \mathbb{L}_{\text {loc }}^{1}\left(\mathbb{R} ; \mathbb{R}^{N}\right)$ with Tot.Var. $\{\bar{u}\}<\delta_{0}$, $\lim _{x \rightarrow-\infty} \bar{u}(x) \in K$, consider the vanishing viscosity solution $u(t, \cdot)$ of the Cauchy problem (1.1), (1.6) (obtained as the unique limit of solutions to the Cauchy problem $(1.3)_{\mu},(1.6)$ when $\left.\mu \rightarrow 0\right)$. Let $u^{\varepsilon}$ be a Glimm approximate solution of (1.1), (1.6), with mesh sizes $\Delta x=\Delta t=\varepsilon$, generated by a sampling sequence $\left\{\theta_{k}\right\}_{k \in \mathbb{N}} \subset[0,1]$ satisfying (1.12). Then, for every $T \geq 0$ there holds

$$
\lim _{\varepsilon \rightarrow 0} \frac{\left\|u^{\varepsilon}(T, \cdot)-u(T, \cdot)\right\|_{\mathbb{L}^{1}}}{\sqrt{\varepsilon}|\log \varepsilon|},
$$

and the limit is uniform w.r.t. $\bar{u}$ as long as Tot.Var. $\{\bar{u}\}<\delta_{0}, \lim _{x \rightarrow-\infty} \bar{u}(x) \in K$.

The proof of the error bound (1.15) follows the same strategy adopted in [12, 5,17 , relying on the careful analysis of the structure of solutions to NGNL systems developed by T.P. Liu and T. Yang in [24,25]. Indeed, to estimate the distance between a Lipschitz continuous (in time) approximate solutions $\psi$ of (1.1) and the corresponding exact solution one would like to use the error bound [11]

$$
\left\|\psi(T)-S_{t} \psi(0)\right\|_{\mathbb{L}^{1}} \leq L \int_{0}^{T} \liminf _{h \rightarrow 0+} \frac{\left\|\psi(t+h)-S_{h} \psi(t)\right\|_{\mathbb{L}^{1}}}{h} d t,
$$

where $L$ denotes a Lipschitz constant of the semigroup $S$ generated by (1.4). However, for approximate solutions constructed by the Glimm scheme, a direct application of this formula is of little help because of the additional errors introduced by the restarting procedures at times $t_{i} \doteq i \varepsilon$. For this reason, following the wave tracing analysis in [25], it is useful to partition the elementary waves present in the approximate solution, say in a time interval $\left[\tau_{1}, \tau_{2}\right]$, into virtual subwaves that can be either traced back from $\tau_{2}$ to $\tau_{1}$ (primary waves), or are canceled or generated by interactions occurring in $\left[\tau_{1}, \tau_{2}\right]$ (secondary waves). Thanks to the simplified wave pattern associated to this partition, one can construct a front tracking approximation having the same initial and terminal values as the Glimm approximation, and thus establish (1.15) relying on (1.16).

The key step of this procedure is to show that the variation of a Glimm functional provides a bound for the change in strength and for the product of strength times the variation in speeds of the primary waves. Here we shall implement a wave tracing algorithm for a general quasilinear system (1.1) in which such bounds are obtained relying on a new interaction potential functional whose decrease at interactions is precisely of the same order of this type of errors. 
To motivate the definition of this functional, consider an interaction between two shock waves of a $k$-th NGNL family, say $s^{\prime}, s^{\prime \prime}$, with speeds $\lambda^{\prime}, \lambda^{\prime \prime}$, respectively, and assume that $s^{\prime}, s^{\prime \prime}$ have the same sign. Then, letting $\lambda$ denote the shock speed of the outgoing wave of the $k$-th family, by the interaction estimates in [7, Theorem 3.7] there holds

$$
[s \Delta \lambda] \doteq\left|s^{\prime}\right|\left|\lambda-\lambda^{\prime}\right|+\left|s^{\prime \prime}\right|\left|\lambda-\lambda^{\prime \prime}\right|=\mathcal{O}(1) \cdot \frac{\left|s^{\prime} s^{\prime \prime}\right|\left|\lambda^{\prime}-\lambda^{\prime \prime}\right|}{\left|s^{\prime}+s^{\prime \prime}\right|} .
$$

Notice that, using the wave-speed maps $\sigma^{\prime}(\cdot), \sigma^{\prime \prime}(\cdot)$ associated to the waves $s^{\prime}, s^{\prime \prime}$ (cfr. Theorem 2), one can rewrite the term on the right-hand side of (1.17) as

$$
\Im\left(s^{\prime}, s^{\prime \prime}\right) \doteq \frac{1}{\left|s^{\prime}\right|+\left|s^{\prime \prime}\right|} \cdot \int_{0}^{\left|s^{\prime}\right|} \int_{0}^{\left|s^{\prime \prime}\right|}\left|\sigma^{\prime}(\xi)-\sigma^{\prime \prime}\left(\xi^{\prime}\right)\right| d \xi d \xi^{\prime} .
$$

Thus, a natural suggestion of the above estimate would be to define the cubic part of a Glimm functional related to the potential interaction of waves of the same family as the sum of terms as (1.18) corresponding to all pair of waves $s^{\prime}, s^{\prime \prime}$ of each characteristic family. In fact, in the present paper we shall consider a Glimm functional defined by

$$
Q(t) \doteq c \cdot \sum_{\substack{k_{\alpha}<k_{\beta} \\ x_{\alpha}(t)>x_{\beta}(t)}}\left|s_{\alpha} s_{\beta}\right|+\sum_{k_{\alpha}=k_{\beta}} \Im\left(s_{\alpha}, s_{\beta}\right)
$$

where, as usual, $x_{\alpha}(t)$ denotes the position of the wave $s_{\alpha}$ in the approximate solution $u^{\varepsilon}(t)$, and $k_{\alpha}$ its characteristic family, while the second summation extends to all pair of waves $s_{\alpha}, s_{\beta}$ of the $k_{\alpha} \in\{1, \ldots, N\}$ family (including $s_{\alpha}=s_{\beta}$ ). Our main result here shows that $Q$ is actually decreasing in time (for a suitable choice of the constant $c>0$ ) at any interaction, and that the products $[s \Delta \lambda]$ of strength times the variation in speeds of the primary waves are bounded by $\mathcal{O}(1) \cdot|\Delta Q|$. Notice that, in the genuinely nonlinear case, the following bounds hold

$$
\frac{1}{\mathcal{O}(1)} \cdot\left|s_{\alpha} s_{\beta}\right| \leq \Im\left(s_{\alpha}, s_{\beta}\right) \leq \mathcal{O}(1) \cdot\left|s_{\alpha} s_{\beta}\right|,
$$

and thus one recovers from (1.19) the standard quadratic interaction potential of the original Glimm functional [16], with the only difference from [16] that in (1.19) all waves of the same family are considered as approaching (even pairs of rarefaction fans).

We conclude recalling that for NGNL systems several Glimm type functionals are available in the literature $[24,25,19,7]$, which work perfectly to establish uniform a-priori bounds on the total variation of the solution, but are not truly effective to control the type of errors $[s \Delta \lambda]$ arising in a wave tracing analysis of the Glimm scheme. On the other hand, in the case of systems satisfying the assumption $(\mathrm{H})$, were recently introduced in $[5,17]$ two type of potential interaction functionals whose decrease actually bounds the products of strength times the variation in speeds $[s \Delta \lambda]$, and which inspired the new definition in (1.19). 
The Glimm functional defined in [5] is the sum of a quadratic term $Q_{q}$ and of the cubic interaction potential defined in [7] concerning waves of the same family, that takes the form $\mathcal{Q}=\sum_{k_{\alpha}=k_{\beta}} \int_{0}^{\left|s_{\alpha}\right|} \int_{0}^{\left|s_{\beta}\right|}\left|\sigma_{\alpha}(\xi)-\sigma_{\beta}\left(\xi^{\prime}\right)\right| d \xi d \xi^{\prime}$. Here, in presence of interactions between waves of the same families and strength smaller than some threshold parameter $\widehat{\delta}, Q_{q}$ behaves as the interaction functional introduced in [3] for systems with a single connected hypersurface (1.13), while the decrease of $\mathcal{Q}$ controls the possible increase of $Q_{q}$ at interactions involving waves of the same family and strength larger than $\widehat{\delta}$. The cubic part of the functional proposed in [17] corresponding to waves of the same family instead depends globally on the wave patterns of the solution. It is defined as $\sum_{k_{\alpha}=k_{\beta}}\left(\left|s_{\alpha}, s_{\beta}\right|\left[\Theta\left(s_{\alpha}, s_{\beta}\right)\right]^{-}\right) / V_{k_{\alpha}}\left(s_{\alpha}, s_{\beta}\right)$, where $\Theta\left(s_{\alpha}, s_{\beta}\right)$ represents the effective angle between $s_{\alpha}$ and $s_{\beta}$, computed taking into account all the $k_{\alpha}$-waves lying between $s_{\alpha}$ and $s_{\beta},[\cdot]^{-}$denotes the negative part, while $V_{k_{\alpha}}\left(s_{\alpha}, s_{\beta}\right)$ is the total strength of all $k_{\alpha}$-waves between $s_{\alpha}$ and $s_{\beta}$ (including $s_{\alpha}$ and $s_{\beta}$ ). Employing these interaction potentials it is shown in $[5,17]$ that, for systems (1.1) satisfying the assumption $(\mathrm{H})$, one can produce a simplified wave partition pattern whose errors are controlled by the total decrease of the corresponding Glimm functional in the time interval taken in consideration, and thus yield the error estimate (1.15). Unfortunately, the decreasing properties of both functionals strongly rely on the assumption that the linearly degenerate manifold (1.13) be a finite union of hypersurfaces transversal to the characteristic vector fields, and thus are of no use to establish an accurate convergence rate for general systems (1.1). Instead, the interaction potential in (1.19) can be applied to a general quasilinear system (1.1), without any assumption on the matrix $A(u)$ a part from the strict hyperbolicity.

\section{Preliminaries}

Let $A$ be a smooth matrix-valued map defined on a domain $\Omega \subset \mathbb{R}^{N}$, with values in the set of $N \times N$ matrices. Assume that each $A(u)$ is strictly hyperbolic and denote by $\left\{\lambda_{1}(u), \ldots, \lambda_{N}(u)\right\} \subset[0,1]$ its eigenvalues. Since we will consider only solutions with small total variation that take values in a neighborhood of a compact set $K \subset \Omega$, it is not restrictive to assume that $\Omega$ is bounded and that there exist constants $\widehat{\lambda}_{0}<\cdots<\widehat{\lambda}_{N}$ such that

$$
\widehat{\lambda}_{k-1}<\lambda_{k}(u)<\hat{\lambda}_{k}, \quad \forall u, \quad k=1, \ldots, N .
$$

One can choose bases of right and left eigenvectors $r_{k}(u), l_{k}(u),(k=1, \ldots, N)$, associated to $\lambda_{k}(u)$, normalized so that

$$
\left|r_{k}(u)\right| \equiv 1, \quad\left\langle l_{h}(u), r_{k}(u)\right\rangle=\left\{\begin{array}{ll}
1 & \text { if } \quad k=h, \\
0 & \text { if } \quad k \neq h,
\end{array} \quad \forall u\right.
$$

By the strict hyperbolicity of the system, in the conservative case (1.4) (where $A(u)=D F(u)$ ), for every fixed $u_{0} \in \Omega$ and for each $k \in\{1, \ldots, N\}$-th characteristic family one can construct in a neighborhood of $u_{0}$ a one-parameter 
smooth curve $S_{k}\left[u_{0}\right]$ passing through $u_{0}$ (called the $k$-th Hugoniot curve issuing from $u_{0}$ ), whose points $u \in S_{k}\left[u_{0}\right]$ satisfy the Rankine Hugoniot equation $F(u)-F\left(u_{0}\right)=\sigma\left(u-u_{0}\right)$ for some scalar $\sigma=\sigma_{k}\left[u_{0}, u\right]$. The curve $S_{k}\left[u_{0}\right]$ is tangent at $u_{0}$ to the right eigenvector $r_{k}\left(u_{0}\right)$ of $A\left(u_{0}\right)$ associated to $\lambda_{k}\left(u_{0}\right)$, and we say that $\left(u^{L}, u^{R}\right)$ is a shock discontinuity of the $k$-th family with speed $\sigma_{k}\left[u^{L}, u^{R}\right]$ if $u^{R} \in S_{k}\left[u^{L}\right]$.

We describe here the general method introduced in $[9,6]$ to construct the self-similar solution of a Riemann problem for a strictly hyperbolic quasilinear system (1.1). As customary, the basic step consists in constructing the elementary curve of the $k$-th family $(k=1, \ldots, N)$ for every given left state $u^{L}$, which is a one parameter curve of right states $s \mapsto T_{k}\left[u^{L}\right](s)$ with the property that the Riemann problem having initial data $\left(u^{L}, u^{R}\right), u^{R} \doteq T_{k}\left[u^{L}\right](s)$, admits a vanishing viscosity solution consisting only of elementary waves of the $k$-th characteristic family. Such a curve is constructed by looking at the fixed point of a suitable contractive transformation associated to a smooth manifold of viscous traveling profiles for the parabolic system with unit viscosity $(1.3)_{1}$.

Given a fixed state $u_{0} \in \Omega$, and an index $k \in\{1, \ldots, N\}$, in connection with the $N+2$-dimensional smooth manifold of bounded traveling profiles of $(1.3)_{1}$ with speed close to $\lambda_{k}\left(u_{0}\right)$, one can define on a neighborhood of $\left(u_{0}, 0, \lambda_{k}\left(u_{0}\right)\right) \in$ $\mathbb{R}^{N} \times \mathbb{R} \times \mathbb{R}$ suitable smooth vector functions $\left(u, v_{k}, \sigma\right) \mapsto \widetilde{r}_{k}\left(u, v_{k}, \sigma\right)$ that satisfy $\widetilde{r}_{k}\left(u_{0}, 0, \sigma\right)=r_{k}\left(u_{0}\right)$, for all $\sigma$, and are normalized so that

$$
\left\langle l_{k}\left(u_{0}\right), \widetilde{r}_{k}\left(u, v_{k}, \sigma\right)\right\rangle=1 \quad \forall u, v_{k}, \sigma .
$$

The vector valued map $\widetilde{r}_{k}\left(u, v_{k}, \sigma\right)$ is called the $k$-th generalized eigenvector of the matrix $A(u)$, associated to the generalized eigenvalue $\widetilde{\lambda}_{k}\left(u, v_{k}, \sigma\right) \doteq$ $\left\langle l_{k}\left(u_{0}\right), A(u) \widetilde{r}_{k}\left(u, v_{k}, \sigma\right)\right\rangle$, that satisfies the identity $\widetilde{\lambda}_{k}\left(u_{0}, v_{k}, \sigma\right)=\lambda_{k}\left(u_{0}\right)$, for all $v_{k}, \sigma$, and, moreover

$$
\frac{\partial}{\partial v_{k}} \widetilde{\lambda}_{k}\left(u, v_{k}, \sigma\right)=\mathcal{O}(1) \cdot\left|u-u_{0}\right|, \quad \frac{\partial}{\partial \sigma} \widetilde{\lambda}_{k}\left(u, v_{k}, \sigma\right)=\mathcal{O}(1) \cdot\left|v_{k}\right|\left|u-u_{0}\right| .
$$

Next, given a left state $u^{L}$ in a neighborhood of $u_{0}$ and $0<s<<1$, consider the integral system

$$
\left\{\begin{array}{l}
u(\tau)=u^{L}+\int_{0}^{\tau} \widetilde{r}_{k}\left(u(\xi), v_{k}(\xi), \sigma(\xi)\right) d \xi \\
v_{k}(\tau)=\widetilde{F}_{k}\left(\tau ; u, v_{k}, \sigma\right)-\operatorname{conv}_{[0, s]} \widetilde{F}_{k}\left(\tau ; u, v_{k}, \sigma\right), \quad 0 \leq \tau \leq s, \\
\sigma(\tau)=\frac{d}{d \tau} \operatorname{conv}_{[0, s]} \widetilde{F}_{k}\left(\tau ; u, v_{k}, \sigma\right),
\end{array}\right.
$$

where $\tau \mapsto \widetilde{f}_{k}(\tau) \doteq \widetilde{F}_{k}\left(\tau ; u, v_{k}, \sigma\right)$ is the "reduced flux function" associated to $(1.3)$, defined by

$$
\widetilde{f}_{k}(\tau) \doteq \int_{0}^{\tau} \widetilde{\lambda}_{k}\left(u(\xi), v_{k}(\xi), \sigma(\xi)\right) d \xi,
$$


and we let $\operatorname{conv}_{[0, s]} \widetilde{f}_{k}(\tau)$ denote the lower convex envelope of $\widetilde{f}_{k}$ on $[0, s]$, i.e.

$$
\begin{aligned}
& \operatorname{conv}_{[0, s]} \tilde{f}_{k}(\tau) \doteq \inf \left\{\theta \widetilde{f}_{k}(y)+(1-\theta) \tilde{f}_{k}(z):\right. \\
& \quad \theta \in[0,1], y, z \in[0, s], \tau=\theta y+(1-\theta) z\} .
\end{aligned}
$$

It is shown in $[9,6]$ that, for $s$ sufficiently small, the transformation $\mathcal{T}_{u^{L}, s}$ defined by the right-hand side of (2.5) maps a domain of continuous curves $\tau \mapsto\left(u(\tau), v_{k}(\tau), \sigma(\tau)\right)$ into itself, and is a contraction w.r.t. a suitable weighted norm. Hence, for every $u^{L}$ in a neighborhood of $u_{0}, s$ in a right neighborhood of zero, the transformation $\mathcal{T}_{u^{L}, s}$ admits a unique fixed point

$$
\tau \mapsto\left(u\left(\tau ; u^{L}, s\right), v_{k}\left(\tau ; u^{L}, s\right), \sigma\left(\tau ; u^{L}, s\right)\right) \quad \tau \in[0, s],
$$

which provides a Lipschitz continuous solution to the integral system (2.5). The elementary curve of right states of the $k$-th family issuing from $u^{L}$ is then defined as the terminal value at $\tau=s$ of the $u$-component of the solution to the integral system (2.5), i.e. by setting

$$
T_{k}\left[u^{L}\right](s) \doteq u\left(s ; u^{L}, s\right) .
$$

Sometimes, the value (2.8) of the elementary curve issuing from $u^{L}$ will be equivalently written $T_{k}(s)\left[u^{L}\right]$. In the following it will be convenient to adopt the notations

$$
\begin{array}{ll}
\sigma_{k}\left[u^{L}\right](s, \tau) & \doteq \sigma\left(\tau ; u^{L}, s\right) \\
\widetilde{F}_{k}\left[u^{L}\right](s, \tau) & \doteq \widetilde{F}_{k}\left(\tau ; u\left(\cdot ; u^{L}, s\right), v_{k}\left(\cdot ; u^{L}, s\right), \sigma\left(\cdot ; u^{L}, s\right)\right)
\end{array} \quad \forall \tau \in[0, s],
$$

for the $\sigma$-component of the solution to (2.5), and for the reduced flux evaluated in connection with such a solution. Notice that by construction the maps $\left(u^{L}, s\right) \mapsto \sigma_{k}\left[u^{L}\right](s, \cdot),\left(u^{L}, s\right) \mapsto \widetilde{F}_{k}\left[u^{L}\right](s, \cdot)$, and the derivative $\left(u^{L}, s\right) \mapsto D_{\tau} \widetilde{F}_{k}\left[u^{L}\right](s, \cdot)$ are Lipschitz continuous for $u^{L}$ in a neighborhood of $u_{0}$, and $s$ in a right neighborhood of zero.

For negative values $s<0,|s|<<1$, one replaces in (2.5) the lower convex envelope of $\widetilde{F}_{k}$ on the interval $[0, s]$ with its upper concave envelope on $[s, 0]$ (defined in analogous way as (2.7)), and then constructs the curve $T_{k}\left[u^{L}\right]$ and the map $\sigma_{k}\left[u^{L}\right]$ exactly in the same way as above looking at the solution of the integral system $(2.5)$ on the interval $[s, 0]$. The elementary curve $T_{k}\left[u^{L}\right]$ and the wave-speed map $\sigma_{k}\left[u^{L}\right]$ constructed in this way enjoy the properties stated in in the following theorem, where we let $\mathcal{C}_{I}([a, b])\left(\mathcal{C}_{D}([b, a])\right)$ denote the set of continuous and increasing (decreasing) scalar functions defined on an interval $[a, b]$, and we set $\mathcal{C}_{I}([a, b]) \doteq \mathcal{C}_{D}([b, a])$ in the case $a>b$.

Theorem $2([\mathbf{9}, \mathbf{6}])$. Let $A$ be a smooth, matrix valued map defined from a domain $\Omega \subset \mathbb{R}^{N}$ into $\mathbf{M}^{N \times N}(\mathbb{R})$, and assume that the matrices $A(u)$ are strictly hyperbolic. Then, for every $u \in \Omega$, there exist $N$ Lipschitz continuous curves 
$s \rightarrow T_{k}[u](s) \in \Omega$ satisfying $\lim _{s \rightarrow 0} \frac{d}{d s} T_{k}[u](s)=r_{k}(u)$, together with $N$ continuous functions $s \rightarrow \sigma_{k}[u](s, \cdot) \in \mathcal{C}_{I}([0, s])(k=1, \ldots, N)$, defined on a neighborhood of zero, so that the following holds. Whenever $u^{L} \in \Omega, u^{R}=T_{k}\left[u^{L}\right](s)$, for some $s$, letting $\mathcal{I} \doteq\left\{\tau \in[0, s]: \sigma_{k}\left[u^{L}\right](s, \tau) \neq \sigma_{k}\left[u^{L}\right]\left(s, \tau^{\prime}\right)\right.$ for all $\left.\tau^{\prime} \neq \tau\right\}$, the piecewise continuous function

$$
u(t, x) \doteq\left\{\begin{array}{ll}
u^{L} & \text { if } x / t<\sigma_{k}\left[u^{L}\right](s, 0), \\
T_{k}\left[u^{L}\right](\tau) & \text { if } x / t=\sigma_{k}\left[u^{L}\right](s, \tau) \\
u^{R} & \text { if } x / t>\sigma_{k}\left[u^{L}\right](s, s),
\end{array} \text { for some } \tau \in \mathcal{I},\right.
$$

provides the unique vanishing viscosity solution (determined by the parabolic approximation (1.3)) of the Riemann problem (1.1), (1.9).

Remark 1. If the system (1.1) is in conservation form, i.e. in the case where $A(u)=D F(u)$ for some smooth flux function $F$, and if the characteristic fields satisfy the assumption $(\mathrm{H})$, the general solution of the Riemann problem provided by (2.10) is a composed wave of the $k$-th family made of a finite number of contact-discontinuities (which satisfy the Liu admissibility condition of Definition 1) adjacent to rarefaction waves. Namely, the regions where the $v_{k}$-component of the solution to (2.5) vanishes correspond to rarefaction waves if the $\sigma$-component is strictly increasing and to contact discontinuities if the $\sigma$-component is constant, while the regions where the $v_{k}$-component of the solution to (2.5) is different from zero correspond to contact discontinuities or to compressive shocks. In particular, whenever the solution of a Riemann problem with initial data $u^{L}, u^{R}=T_{k}\left[u^{L}\right](s)$ contains a Liu admissible shock joining, say, two states $T_{k}\left[u^{L}\right]\left(s^{\prime}\right), T_{k}\left[u^{L}\right]\left(s^{\prime \prime}\right), s^{\prime}, s^{\prime \prime} \in[0, s]$, one has $\sigma_{k}\left[u^{L}\right]\left(s, s^{\prime}\right)=$ $\sigma_{k}\left[u^{L}\right](s, \tau)$ for all $\tau \in\left[s^{\prime}, s^{\prime \prime}\right]$, and $\sigma_{k}\left[u^{L}\right]\left(s, s^{\prime}\right)$ provides the shock speed of the discontinuity $\left(T_{k}\left[u^{L}\right]\left(s^{\prime}\right), T_{k}\left[u^{L}\right]\left(s^{\prime \prime}\right)\right)$. Clearly, in a non conservative setting, "admissibility" for a jump means precisely that the jump corresponds to a traveling profile for the parabolic approximation $(1.3)_{1}$.

Once we have constructed the elementary curves $T_{k}$ for each $k$-th characteristic family, the vanishing viscosity solution of a general Riemann problem for (1.4) is then obtained by a standard procedure observing that the composite mapping

$$
\left(s_{1}, \ldots, s_{N}\right) \mapsto T_{N}\left(s_{N}\right) \circ \cdots \circ T_{1}\left(s_{1}\right)\left[u^{L}\right] \doteq u^{R},
$$

is one-to-one from a neighborhood of the origin in $\mathbb{R}^{N}$ onto a neighborhood of $u^{L}$. This is a consequence of the fact that the curves $T_{k}[u]$ are tangent to $r_{k}(u)$ at zero (cfr. Theorem 2), and then follows by applying a version of the implicit function theorem valid for Lipschitz continuous maps. Therefore, we can uniquely determine intermediate states $u^{L} \doteq \omega_{0}, \omega_{1}, \ldots, \omega_{N} \doteq u^{R}$, and wave sizes $s_{1}, \ldots, s_{N}$, such that there holds

$$
\omega_{k}=T_{k}\left[\omega_{k-1}\right]\left(s_{k}\right) \quad k=1, \ldots, N,
$$


provided that the left and right states $u^{L}, u^{R}$ are sufficiently close to each other. Each Riemann problem with initial datum

$$
\bar{u}_{k}(x)=\left\{\begin{array}{lll}
\omega_{k-1} & \text { if } & x<0 \\
\omega_{k} & \text { if } & x>0
\end{array}\right.
$$

admits a vanishing viscosity solution of total size $s_{k}$, containing a sequence of rarefactions and Liu admissible discontinuities of the $k$-th family. Then, because of the uniform strict hyperbolicity assumption (2.1), the general solution of the Riemann Problem with initial data $\left(u^{L}, u^{R}\right)$ is obtained by piecing together the vanishing viscosity solutions of the elementary Riemann problems (1.4) (2.13). Throughout the paper, with a slight abuse of notation, we shall often call $s$ a wave of (total) size $s$, and, if $u^{R}=T_{k}\left[u^{L}\right](s)$, we will say that $\left(u^{L}, u^{R}\right)$ is a wave of size $s$ of the $k$-th characteristic family.

A fundamental ingredient to establish an accurate convergence rate for the Glimm scheme is the wave tracing procedure, which was first introduced by T.P. Liu in his celebrated paper [21] for systems with genuinely nonlinear or linearly degenerate fields, and lately extended to systems fulfilling assumption $(\mathrm{H})[24,25]$. In this spirit, we have introduced in [5] the following notion of partition of a $k$-wave $\left(u^{L}, u^{R}\right)$, defined in terms of the elementary curves $T_{k}$ at (2.8)

Definition 2. Given a pair of states $u^{L}$, $u^{R}$, with $u^{R}=T_{k}\left[u^{L}\right](s)$ for some $s>0$, we say that a set $\left\{y^{1}, \ldots, y^{\ell}\right\}$ is a partition of the $k$-th wave $\left(u^{L}, u^{R}\right)$ at time $i \varepsilon$, if the followings holds.

1. There exist scalars $s^{h}>0, h=1, \ldots, l$, such that, setting $\tau^{h} \doteq \sum_{p=1}^{h} s^{p}$, $w^{h} \doteq T_{k}\left[u^{L}\right]\left(\tau^{h}\right)$, there holds

$$
y^{h}=w^{h}-w^{h-1} \quad \forall h .
$$

The quantity $s^{h}$ is called the size of the elementary wave $y^{h}$.

2. Letting $\sigma \doteq \sigma_{k}\left[u^{L}\right](s, \cdot)$ be the map in (2.9), there holds

$$
\sigma\left(s_{h}\right)-\sigma\left(s_{h-1}\right) \leq \varepsilon \quad \forall h .
$$

Moreover, we require that $\left.\theta_{i+1} \notin\right] \sigma\left(\tau^{h-1}\right), \sigma\left(\tau^{h}\right)[$, for all $h$ (so to avoid further partitions of $y^{h}$ at $\left.t=(i+1) \varepsilon\right)$.

The definition is entirely similar in the case $u^{R}=T_{k}\left[u^{L}\right](s)$, with $s<0$. In connection with a partition $\left\{y^{1}, \ldots, y^{\ell}\right\}$ of $\left(u^{L}, u^{R}\right)$, we define the corresponding speed of the elementary wave $y^{h}$ as

$$
\lambda_{k}^{h} \doteq \frac{1}{s^{h}} \int_{\tau^{h-1}}^{\tau^{h}} \sigma(\tau) d \tau \quad \forall h .
$$

We conclude the section providing the following definition of quantity of interaction introduced in [7, Definition 3.5] for a general strictly hyperbolic system (1.1), which is useful to measure the decrease of the functional $Q$ in (1.19) when waves of the same family interact together. 
Definition 3. Consider two waves of sizes $s^{\prime}, s^{\prime \prime}$, belonging to the the same $k$-th characteristic family, with left states $u^{\prime}, u^{\prime \prime}$, respectively. Let $\widetilde{F}^{\prime} \doteq \widetilde{F}_{k}\left[u^{\prime}\right]\left(s^{\prime}, \cdot\right)$ and $\widetilde{F}^{\prime \prime} \doteq \widetilde{F}_{k}\left[u^{\prime \prime}\right]\left(s^{\prime \prime}, \cdot\right)$ be the reduced flux with starting point $u^{\prime}, u^{\prime \prime}$, evaluated along the solution of $(2.5)$ on the interval $\left[0, s^{\prime}\right]$, and $\left[0, s^{\prime \prime}\right]$, respectively (cfr. def. (2.9)). Then, assuming that $s^{\prime} \geq 0$, we say that the amount of interaction $\mathcal{J}\left(s^{\prime}, s^{\prime \prime}\right)$ between $s^{\prime}$ and $s^{\prime \prime}$ is the quantity defined as follows.

1. If $s^{\prime \prime} \geq 0$ set:

$$
\begin{aligned}
& \mathcal{J}\left(s^{\prime}, s^{\prime \prime}\right) \doteq \int_{0}^{s^{\prime}}\left|\operatorname{conv}_{\left[0, s^{\prime}\right]} \widetilde{F}^{\prime}(\xi)-\operatorname{conv}_{\left[0, s^{\prime}+s^{\prime \prime}\right]} \widetilde{F}^{\prime} \cup \widetilde{F}^{\prime \prime}(\xi)\right| d \xi \\
& \quad+\int_{s^{\prime}}^{s^{\prime}+s^{\prime \prime}}\left|\widetilde{F}^{\prime}\left(s^{\prime}\right)+\operatorname{conv}_{\left[0, s^{\prime \prime}\right]} \widetilde{F}^{\prime \prime}\left(\xi-s^{\prime}\right)-\operatorname{conv}_{\left[0, s^{\prime}+s^{\prime \prime}\right]} \widetilde{F}^{\prime} \cup \widetilde{F}^{\prime \prime}(\xi)\right| d \xi,
\end{aligned}
$$

where $\widetilde{F}^{\prime} \cup \widetilde{F}^{\prime \prime}$ is the function defined on $\left[0, s^{\prime}+s^{\prime \prime}\right]$ as

$$
\widetilde{F}^{\prime} \cup \widetilde{F}^{\prime \prime}(s) \doteq \begin{cases}\widetilde{F}^{\prime}(s) & \text { if } \quad s \in\left[0, s^{\prime}\right], \\ \widetilde{F}^{\prime}\left(s^{\prime}\right)+\widetilde{F}^{\prime \prime}\left(s-s^{\prime}\right) & \text { if } s \in\left[s^{\prime}, s^{\prime}+s^{\prime \prime}\right] .\end{cases}
$$

2. If $-s^{\prime} \leq s^{\prime \prime}<0$ set:

$$
\begin{aligned}
\mathcal{J}\left(s^{\prime}, s^{\prime \prime}\right) & \doteq \int_{0}^{s^{\prime}+s^{\prime \prime}}\left|\operatorname{conv}_{\left[0, s^{\prime}\right]} \widetilde{F}^{\prime}(\xi)-\operatorname{conv}_{\left[0, s^{\prime}+s^{\prime \prime}\right]} \widetilde{F}^{\prime}(\xi)\right| d \xi \\
& +\int_{s^{\prime}+s^{\prime \prime}}^{s^{\prime}}\left|\operatorname{conv}_{\left[0, s^{\prime}\right]} \widetilde{F}^{\prime}(\xi)-\operatorname{conc}_{\left[s^{\prime}+s^{\prime \prime}, s^{\prime}\right]} F^{\prime}(\xi)\right| d \xi .
\end{aligned}
$$

3. If $s^{\prime \prime}<-s^{\prime}$ set:

$$
\begin{aligned}
\mathcal{J}\left(s^{\prime}, s^{\prime \prime}\right) \doteq & \int_{s^{\prime}+s^{\prime \prime}}^{0}\left|\operatorname{conc}_{\left[s^{\prime \prime}, 0\right]} \widetilde{F}^{\prime \prime}\left(\xi-s^{\prime}\right)-\operatorname{conc}_{\left[s^{\prime \prime},-s^{\prime}\right]} \widetilde{F}^{\prime \prime}\left(\xi-s^{\prime}\right)\right| d \xi \\
& +\int_{0}^{s^{\prime}}\left|\operatorname{conc}_{\left[s^{\prime \prime}, 0\right]} \widetilde{F}^{\prime \prime}\left(\xi-s^{\prime}\right)-\operatorname{conv}_{\left[-s^{\prime}, 0\right]} \widetilde{F}^{\prime \prime}\left(\xi-s^{\prime}\right)\right| d \xi
\end{aligned}
$$

Here, $\operatorname{conv}_{[a, b]} f, \operatorname{conc}_{[a, b]} f$ denote the lower convex envelope and the upper concave envelope of $f$ on $[a, b]$, defined as in (2.7). In the case where $s^{\prime}<0$, one replaces in (2.15)-(2.18) the lower convex envelope with the upper concave one, and vice-versa.

Remark 2. By the Lipschitz continuity of the maps $(u, s) \mapsto \widetilde{F}_{k}\left[u^{L}\right](s, \cdot)$, $(u, s) \mapsto D_{\tau} \widetilde{F}_{k}\left[u^{L}\right](s, \cdot)$ it follows that

$$
\mathcal{J}\left(s^{\prime}, s^{\prime \prime}\right)=\mathcal{O}(1) \cdot\left|s^{\prime} s^{\prime \prime}\right|
$$

Moreover, by Remark 1 one can easily verify that, in the conservative case, if $s^{\prime}, s^{\prime \prime}$ are both shocks of the $k$-th family that have the same sign, then the amount of interaction in (2.15) takes the form

$$
\mathcal{J}\left(s^{\prime}, s^{\prime \prime}\right)=\left|s^{\prime} s^{\prime \prime}\right|\left|\sigma_{k}\left[u^{L}, u^{M}\right]-\sigma_{k}\left[u^{M}, u^{R}\right]\right|,
$$


i.e. it is precisely the product of the strength of the waves times the difference of their Rankine Hugoniot speeds.

\section{New wave interaction potential}

In this section we first collect the basic estimates on the change in size and speed of the elementary waves of an approximate solution constructed by the Glimm scheme, and next establish the a-priori bounds on the decrease of the potential interaction defined in (1.19). To this end, for every given wave $s$ of the $k$-th family, set

$$
\Sigma(s) \doteq \int_{0}^{s} \sigma(\tau) d \tau
$$

where $\sigma(\cdot) \doteq \sigma_{k}[w](s, \cdot)$ is the wave-speed map in $(2.9)$, with $w$ being the left state of $s$. Then, relying on the analysis in [7, Section 3] of the effect of wave interaction on the solution of Riemann problems for general quasilinear systems (1.1), we derive the following

Lemma 1. For every compact set $K \subset \Omega$, there exists a constant $\chi_{1}>0$ such that the following holds. Let $s_{1}^{\prime}, \ldots, s_{N}^{\prime}$ and $s_{1}^{\prime \prime}, \ldots, s_{N}^{\prime \prime}$ be, respectively, the sizes of the waves in the solution of two adjacent Riemann problems $\left(u^{L}, u^{M}\right)$ and $\left(u^{M}, u^{R}\right), s_{i}^{\prime}$ and $s_{i}^{\prime \prime}$ belonging to the $i$-th characteristic family, with $u^{L}, u^{M}, u^{R} \in K$, and $\left|s_{i}^{\prime}\right|,\left|s_{i}^{\prime \prime}\right| \leq \chi_{1}$ for all $i=1, \ldots, N$. Call $s_{1}, \ldots, s_{N}$ the sizes of the waves in the solution of the Riemann problem $\left(u^{L}, u^{R}\right), s_{i}$ belonging to the $i$-th characteristic family. Then, there holds

$$
\begin{aligned}
\sum_{k=1}^{N}\left|s_{k}-s_{k}^{\prime}-s_{k}^{\prime \prime}\right| & =\mathcal{O}(1) \cdot\left[\sum_{\substack{1 \leq i, j \leq N \\
i>j}}\left|s_{i}^{\prime} s_{j}^{\prime \prime}\right|+\sum_{1 \leq i \leq N} \mathcal{J}\left(s_{i}^{\prime}, s_{i}^{\prime \prime}\right)\right], \\
\sum_{k=1}^{N}\left|\Sigma\left(s_{k}\right)-\Sigma\left(s_{k}^{\prime}\right)-\Sigma\left(s_{k}^{\prime \prime}\right)\right| & =\mathcal{O}(1) \cdot\left[\sum_{\substack{1 \leq i, j \leq N \\
i>j}}\left|s_{i}^{\prime} s_{j}^{\prime \prime}\right|+\sum_{1 \leq i \leq N} \mathcal{J}\left(s_{i}^{\prime}, s_{i}^{\prime \prime}\right)\right] .
\end{aligned}
$$

Proof. A proof of the estimate (3.2) can be found in [7], thus we will focus our attention only on (3.3). Notice that, by the analysis in [7, Section 3] it immediately follows that the changes of the quantity $\Sigma$ in (3.1) due to interactions between waves of different families is controlled by the product of the strengths of the approaching waves. Hence, it will be sufficient to establish (3.3) in the case where the two adjacent Riemann problems are both solved by a single wave of the same $k$-th family, $s_{k}^{\prime}$ and $s_{k}^{\prime \prime}\left(s_{k}^{\prime}\right.$ on the left of $\left.s_{k}^{\prime \prime}\right)$. Thus, $u^{L}, u^{M}$ are the states on the left of $s_{k}^{\prime}$ and $s_{k}^{\prime \prime}$ respectively, and $u^{R}$ is the state on the right of $s_{k}^{\prime \prime}$. Call $u^{L_{k}}$ the left state of the outgoing wave of the $k$-th family, $s_{k}$. 
Notice that (3.2) yields

$$
\begin{aligned}
\left|u^{L}-u^{L_{k}}\right| & =\mathcal{O}(1) \cdot \mathcal{J}\left(s_{k}^{\prime}, s_{k}^{\prime \prime}\right), \\
\left|s_{j}\right| & =\mathcal{O}(1) \cdot \mathcal{J}\left(s_{k}^{\prime}, s_{k}^{\prime \prime}\right) \quad \forall j \neq k .
\end{aligned}
$$

Moreover, by (2.5), (2.9) one has

$$
\begin{aligned}
\Sigma\left(s_{k}^{\prime}\right) & =\widetilde{F}_{k}\left[u^{L}\right]\left(s_{k}^{\prime}, s_{k}^{\prime}\right), \\
\Sigma\left(s_{k}^{\prime \prime}\right) & =\widetilde{F}_{k}\left[u^{M}\right]\left(s_{k}^{\prime \prime}, s_{k}^{\prime \prime}\right), \\
\Sigma\left(s_{k}\right) & =\widetilde{F}_{k}\left[u^{L_{k}}\right]\left(s_{k}, s_{k}\right) .
\end{aligned}
$$

Hence, since the Lipschitz continuity of the reduced flux map $u \mapsto \widetilde{F}_{k}[u](s, \cdot)$ at (2.9) implies

$$
\left|\widetilde{F}_{k}\left[u^{L_{k}}\right]\left(s_{k}, s_{k}\right)-\widetilde{F}_{k}\left[u^{L}\right]\left(s_{k}, s_{k}\right)\right|=\mathcal{O}(1) \cdot\left|u^{L_{k}}-u^{L}\right|,
$$

and because

$$
\Sigma\left(s_{j}^{\prime}\right)=\Sigma\left(s_{j}^{\prime \prime}\right)=0 \quad \forall j \neq k,
$$

it follows from (3.4)-(3.6) that in order to establish (3.3) it suffices to prove

$$
\left|\widetilde{F}_{k}\left[u^{L}\right]\left(s_{k}, s_{k}\right)-\widetilde{F}_{k}\left[u^{L}\right]\left(s_{k}^{\prime}, s_{k}^{\prime}\right)-\widetilde{F}_{k}\left[u^{M}\right]\left(s_{k}^{\prime \prime}, s_{k}^{\prime \prime}\right)\right|=\mathcal{O}(1) \cdot \mathcal{J}\left(s_{k}^{\prime}, s_{k}^{\prime \prime}\right) .
$$

We will consider two cases, depending on the sign of $s_{k}^{\prime} \cdot s_{k}^{\prime \prime}$.

Case 1: $s_{k}^{\prime} \cdot s_{k}^{\prime \prime}>0$. For the sake of simplicity, assume that $s_{k} \geq s_{k}^{\prime}+s_{k}^{\prime \prime} \geq 0$.

Recalling the definition of reduced flux at (2.6) and (2.9), we have

$$
\begin{aligned}
\widetilde{F}_{k}\left[u^{L}\right]\left(s_{k}, \tau\right) & =\int_{0}^{\tau} \widetilde{\lambda}_{k}\left(u(\xi), v_{k}(\xi), \sigma(\xi)\right) d \xi, \\
\widetilde{F}_{k}\left[u^{L}\right]\left(s_{k}^{\prime}, \tau\right) & =\int_{0}^{\tau} \widetilde{\lambda}_{k}\left(u^{\prime}(\xi), v_{k}^{\prime}(\xi), \sigma^{\prime}(\xi)\right) d \xi, \\
\widetilde{F}_{k}\left[u^{M}\right]\left(s_{k}^{\prime \prime}, \tau\right) & =\int_{0}^{\tau} \widetilde{\lambda}_{k}\left(u^{\prime \prime}(\xi), v_{k}^{\prime \prime}(\xi), \sigma^{\prime \prime}(\xi)\right) d \xi .
\end{aligned}
$$

where

$$
\begin{aligned}
\gamma(\xi) & \doteq\left(u(\xi), v_{k}(\xi), \sigma(\xi)\right), \\
\gamma^{\prime}(\xi) & \doteq\left(u^{\prime}(\xi), v_{k}^{\prime}(\xi), \sigma^{\prime}(\xi)\right), \\
\gamma^{\prime \prime}(\xi) & \doteq\left(u^{\prime \prime}(\xi), v_{k}^{\prime \prime}(\xi), \sigma^{\prime \prime}(\xi)\right),
\end{aligned}
$$

are the solutions of the integral system (2.5) associated to the operators $\mathcal{T}_{u^{L}, s_{k}}, \mathcal{T}_{u^{L}, s_{k}^{\prime}}, \mathcal{T}_{u^{M}, s_{k}^{\prime \prime}}$, defined by the right-hand side of (2.5). Notice that by (3.2) there holds

$$
\begin{aligned}
\int_{s_{k}^{\prime}+s_{k}^{\prime \prime}}^{s_{k}} \tilde{\lambda}_{k}\left(u(\xi), v_{k}(\xi), \sigma(\xi)\right) d \xi & =\mathcal{O}(1) \cdot\left|s_{k}-s_{k}^{\prime}-s_{k}^{\prime \prime}\right| \\
& =\mathcal{O}(1) \cdot \mathcal{J}\left(s_{k}^{\prime}, s_{k}^{\prime \prime}\right),
\end{aligned}
$$


which yields

$$
\begin{aligned}
& \left|\widetilde{F}_{k}\left[u^{L}\right]\left(s_{k}, s_{k}\right)-\widetilde{F}_{k}\left[u^{L}\right]\left(s_{k}^{\prime}, s_{k}^{\prime}\right)-\widetilde{F}_{k}\left[u^{M}\right]\left(s_{k}^{\prime \prime}, s_{k}^{\prime \prime}\right)\right|= \\
& =\mathcal{O}(1) \cdot \mathcal{J}\left(s_{k}^{\prime}, s_{k}^{\prime \prime}\right)+\mid \int_{0}^{s_{k}^{\prime}+s_{k}^{\prime \prime}} \widetilde{\lambda}_{k}\left(u(\xi), v_{k}(\xi), \sigma(\xi)\right) d \xi+ \\
& \quad \quad-\int_{0}^{s_{k}^{\prime}} \widetilde{\lambda}_{k}\left(u^{\prime}(\xi), v_{k}^{\prime}(\xi), \sigma^{\prime}(\xi)\right) d \xi-\int_{0}^{s_{k}^{\prime \prime}} \widetilde{\lambda}_{k}\left(u^{\prime \prime}(\xi), v_{k}^{\prime \prime}(\xi), \sigma^{\prime \prime}(\xi)\right) d \xi \mid \\
& =\mathcal{O}(1) \cdot \mathcal{J}\left(s_{k}^{\prime}, s_{k}^{\prime \prime}\right)+I_{1}+I_{2},
\end{aligned}
$$

where

$$
\begin{aligned}
& I_{1}=\int_{0}^{s_{k}^{\prime}}\left|\widetilde{\lambda}_{k}\left(u(\xi), v_{k}(\xi), \sigma(\xi)\right)-\widetilde{\lambda}_{k}\left(u^{\prime}(\xi), v_{k}^{\prime}(\xi), \sigma^{\prime}(\xi)\right)\right| d \xi \\
& I_{2}=\int_{0}^{s_{k}^{\prime \prime}}\left|\widetilde{\lambda}_{k}\left(u\left(s_{k}^{\prime}+\xi\right), v_{k}\left(s_{k}^{\prime}+\xi\right), \sigma\left(s_{k}^{\prime}+\xi\right)\right)-\widetilde{\lambda}_{k}\left(u^{\prime \prime}(\xi), v_{k}^{\prime \prime}(\xi), \sigma^{\prime \prime}(\xi)\right)\right| d \xi .
\end{aligned}
$$

Setting

$$
\Gamma \doteq\left\{\left(u, v_{k}, \sigma\right):\left|u-u_{0}\right|,\left|v_{k}\right|,|\sigma| \leq \bar{\delta}\right\}
$$

with

$$
\bar{\delta} \doteq \max \left\{\sup _{0 \leq \xi \leq s_{k}}\left|u(\xi)-u_{0}\right|, \sup _{0 \leq \xi \leq s_{k}^{\prime}}\left|u^{\prime}(\xi)-u_{0}\right|\right\},
$$

thanks to (2.4) we may estimate the term $I_{1}$ as

$$
\begin{aligned}
I_{1} \leq \mathcal{O}(1) \cdot \int_{0}^{s_{k}^{\prime}} & {\left[\sup _{\left(u, v_{k}, \sigma\right) \in \Gamma}\left|D_{u} \widetilde{\lambda}_{k}\left(u, v_{k}, \sigma\right)\right| \cdot\left|u(\xi)-u^{\prime}(\xi)\right|+\right.} \\
& +\sup _{\left(u, v_{k}, \sigma\right) \in \Gamma}\left|D_{v_{k}} \widetilde{\lambda}_{k}\left(u, v_{k}, \sigma\right)\right| \cdot\left|v_{k}(\xi)-v_{k}^{\prime}(\xi)\right|+ \\
& \left.+\sup _{\left(u, v_{k}, \sigma\right) \in \Gamma} \frac{\left|D_{\sigma} \widetilde{\lambda}_{k}\left(u, v_{k}, \sigma\right)\right|}{\left|v_{k}\right|} \cdot\left|v_{k}(\xi)\right| \cdot\left|\sigma(\xi)-\sigma^{\prime}(\xi)\right|\right] d \xi \\
\leq \mathcal{O}(1) \cdot \max \{ & \left.s_{k}^{\prime}, \bar{\delta}\right\} \cdot\left[\left\|u-u^{\prime}\right\|_{\infty, s_{k}^{\prime}}+\left\|v_{k}-v_{k}^{\prime}\right\|_{1, s_{k}^{\prime}}+\left\|v_{k} \sigma-v_{k}^{\prime} \sigma^{\prime}\right\|_{1, s_{k}^{\prime}}\right],
\end{aligned}
$$

where $\|\cdot\|_{\infty, s_{k}^{\prime}},\|\cdot\|_{1, s_{k}^{\prime}}$ denote the $\mathbb{L}^{\infty}$ and $\mathbb{L}^{1}$ norm, respectively, relative to the interval $\left[0, s_{k}^{\prime}\right]$. Then, observing that by the proof of [7, Lemma 3.9], (assuming $s_{k}^{\prime}, \bar{\delta}$ sufficiently small) one has

$\max \left\{s_{k}^{\prime}, \bar{\delta}\right\} \cdot\left\|u-u^{\prime}\right\|_{\infty, s_{k}^{\prime}}+\left\|v_{k}-v_{k}^{\prime}\right\|_{1, s_{k}^{\prime}}+\left\|v_{k} \sigma-v_{k}^{\prime} \sigma^{\prime}\right\|_{1, s_{k}^{\prime}}=\mathcal{O}(1) \cdot \mathcal{J}\left(s_{k}^{\prime}, s_{k}^{\prime \prime}\right)$,

from (3.13) we deduce

$$
I_{1}=\mathcal{O}(1) \cdot \mathcal{J}\left(s_{k}^{\prime}, s_{k}^{\prime \prime}\right)
$$


The quantity $I_{2}$ can be estimated in an entirely similar way, so that due to $(3.10)$ we recover $(3.8)$.

Case 2: $s_{k}^{\prime} \cdot s_{k}^{\prime \prime}<0$. To fix the ideas, assume that $s_{k}^{\prime} \geq-s_{k}^{\prime \prime} \geq 0$. As in (3.10), relying on (3.2), (3.9) we derive

$$
\begin{aligned}
\mid \widetilde{F}_{k}\left[u^{L}\right]\left(s_{k}, s_{k}\right)-\widetilde{F}_{k}\left[u^{L}\right]\left(s_{k}^{\prime}, s_{k}^{\prime}\right)- & \widetilde{F}_{k}\left[u^{M}\right]\left(s_{k}^{\prime \prime}, s_{k}^{\prime \prime}\right) \mid= \\
& =\mathcal{O}(1) \cdot \mathcal{J}\left(s_{k}^{\prime}, s_{k}^{\prime \prime}\right)+I_{3}+I_{4},
\end{aligned}
$$

where

$$
\begin{array}{r}
I_{3}=\int_{0}^{s_{k}^{\prime}+s_{k}^{\prime \prime}}\left|\widetilde{\lambda}_{k}\left(u(\xi), v_{k}(\xi), \sigma(\xi)\right)-\widetilde{\lambda}_{k}\left(u^{\prime}(\xi), v_{k}^{\prime}(\xi), \sigma^{\prime}(\xi)\right)\right| d \xi \\
I_{4}=\int_{0}^{-s_{k}^{\prime \prime}} \mid \widetilde{\lambda}_{k}\left(u^{\prime}\left(s_{k}^{\prime}+s_{k}^{\prime \prime}+\xi\right), v_{k}^{\prime}\left(s_{k}^{\prime}+s_{k}^{\prime \prime}+\xi\right), \sigma^{\prime}\left(s_{k}^{\prime}+s_{k}^{\prime \prime}+\xi\right)\right)+ \\
-\widetilde{\lambda}_{k}\left(u^{\prime \prime}\left(s_{k}^{\prime \prime}+\xi\right), v_{k}^{\prime \prime}\left(s_{k}^{\prime \prime}+\xi\right), \sigma^{\prime \prime}\left(s_{k}^{\prime \prime}+\xi\right)\right) \mid d \xi .
\end{array}
$$

The quantities $I_{3}, I_{4}$ can be estimated in an entirely similar way as $I_{1}$ in (3.13)-(3.15) obtaining

$$
I_{3}=\mathcal{O}(1) \cdot \mathcal{J}\left(s_{k}^{\prime}, s_{k}^{\prime \prime}\right), \quad I_{4}=\mathcal{O}(1) \cdot \mathcal{J}\left(s_{k}^{\prime}, s_{k}^{\prime \prime}\right) .
$$

Hence (3.8) follows from (3.16), (3.17), thus completing the proof of the lemma.

As customary, we define the total strength of waves in an approximate solution $u^{\epsilon}(t)$ as

$$
V(t) \doteq \sum_{\alpha}\left|s_{\alpha}\right|
$$

Moreover, for every pair of waves of the same family $s^{\prime}, s^{\prime \prime}$, we define the amount of cancellation as

$$
\mathcal{C}\left(s^{\prime}, s^{\prime \prime}\right) \doteq \begin{cases}\min \left\{\left|s^{\prime}\right|,\left|s^{\prime \prime}\right|\right\} & \text { if } s^{\prime} s^{\prime \prime}<0 \\ 0 & \text { otherwise }\end{cases}
$$

and we introduce the following definitions of quantity of interaction.

Definition 4. Consider two waves of sizes $s^{\prime}, s^{\prime \prime}$ belonging to the the same $k$-th characteristic family, with left states $u^{\prime}, u^{\prime \prime}$, respectively. Let $\sigma^{\prime} \doteq \sigma_{k}\left[u^{\prime}\right]\left(s^{\prime}, \cdot\right)$, $\sigma^{\prime \prime} \doteq \sigma_{k}\left[u^{\prime \prime}\right]\left(s^{\prime \prime}, \cdot\right)$ denote the corresponding wave-speed maps defined in $(2.9)$, and set

$$
\mathcal{I}\left(s^{\prime}, s^{\prime \prime}\right) \doteq \int_{0}^{\left|s^{\prime}\right|} \int_{0}^{\left|s^{\prime \prime}\right|}\left|\sigma^{\prime}(\xi)-\sigma^{\prime \prime}\left(\xi^{\prime}\right)\right| d \xi d \xi^{\prime} .
$$


Next, letting $\widetilde{F}^{\prime} \doteq \widetilde{F}_{k}\left[u^{\prime}\right]\left(s^{\prime}, \cdot\right), \widetilde{F}^{\prime \prime} \doteq \widetilde{F}_{k}\left[u^{\prime \prime}\right]\left(s^{\prime \prime}, \cdot\right)$ be the reduced flux with starting point $u^{\prime}, u^{\prime \prime}$, evaluated along the solution of $(2.5)$ on the interval $\left[0, s^{\prime}\right]$, and $\left[0, s^{\prime \prime}\right]$, respectively (cfr. def. (2.9)), and assuming that $s^{\prime}, s^{\prime \prime} \geq 0$, set

$$
\begin{aligned}
& \tau^{\prime} \doteq \sup \left\{\tau \in\left[0, s^{\prime}\right]: \operatorname{conv}_{\left[0, s^{\prime}\right]} \widetilde{F}^{\prime}(\xi)=\operatorname{conv}_{\left[0, s^{\prime}+s^{\prime \prime}\right]} \widetilde{F}^{\prime} \cup \widetilde{F}^{\prime \prime}(\xi) \quad \forall \xi \in[0, \tau]\right\}, \\
& \tau^{\prime \prime} \doteq \inf \left\{\tau \in\left[0, s^{\prime \prime}\right]: \operatorname{conv}_{\left[0, s^{\prime \prime}\right]} \widetilde{F}^{\prime \prime}(\xi)=\operatorname{conv}_{\left[0, s^{\prime}+s^{\prime \prime}\right]} \widetilde{F}^{\prime} \cup \widetilde{F}^{\prime \prime}(\xi) \quad \forall \xi \in\left[\tau, s^{\prime \prime}\right]\right\},
\end{aligned}
$$

(adopting the same notations of Definition 3). Then, we define the quantity of effective interaction $\mathcal{I}^{e}\left(s^{\prime}, s^{\prime \prime}\right)$ between $s^{\prime}$ and $s^{\prime \prime}$ as

$$
\mathcal{I}^{e}\left(s^{\prime}, s^{\prime \prime}\right) \doteq \mathcal{I}\left(s^{\prime}-\tau^{\prime}, \tau^{\prime \prime}\right),
$$

where the right hand-side of (3.22) is defined according with (3.20) interpreting $s^{\prime}-\tau^{\prime}, \tau^{\prime \prime}$ as waves of the $k$-th family with left states $u^{\sharp} \doteq T_{k}\left[u^{\prime}\right]\left(\tau^{\prime}\right)$ and $u^{\prime \prime}$, respectively. Entirely similar definitions are given when $s^{\prime}, s^{\prime \prime}$ have both negative sign.

Remark 3. By the Lipschitz continuity of the map $(u, s) \mapsto \sigma_{k}[u](s, \cdot)$ it follows that

$$
\mathcal{I}\left(s^{\prime}, s^{\prime \prime}\right)=\mathcal{O}(1) \cdot\left|s^{\prime} s^{\prime \prime}\right| \cdot\left[\left|s^{\prime}\right|+\left|s^{\prime \prime}\right|+\left|u^{\prime}-u^{\prime \prime}\right|\right],
$$

and, in the particular case where $s^{\prime}=s^{\prime \prime}$, one has

$$
\mathcal{I}(s, s)=\mathcal{O}(1) \cdot|s|^{3} .
$$

Remark 4. Employing the definition (3.20) we can rewrite the functional $Q$ introduced in (1.19) as

$$
Q(t) \doteq c \cdot \sum_{\substack{k_{\alpha}<k_{\beta} \\ x_{\alpha}(t)>x_{\beta}(t)}}\left|s_{\alpha} s_{\beta}\right|+\sum_{k_{\alpha}=k_{\beta}} \frac{\mathcal{I}\left(s_{\alpha}, s_{\beta}\right)}{\left|s_{\alpha}\right|+\left|s_{\beta}\right|} .
$$

Remark 5. If $s^{\prime}, s^{\prime \prime}$ are two adjacent waves with the same sign and belonging to the same characteristic family, by the analysis in $[7, \S 4]$ and $[8]$, and because of $[7$, Remark 3.6], it follows

$$
\mathcal{J}\left(s^{\prime}, s^{\prime \prime}\right)=\mathcal{O}(1) \cdot \mathcal{I}^{e}\left(s^{\prime}, s^{\prime \prime}\right) .
$$

Moreover, if we consider the potential interaction functional introduced in [7]

$$
\mathcal{Q}(t) \doteq \sum_{\substack{k_{\alpha}<k_{\beta} \\ x_{\alpha}(t)>x_{\beta}(t)}}\left|s_{\alpha} s_{\beta}\right|+\frac{1}{4} \sum_{k_{\alpha}=k_{\beta}} \mathcal{I}\left(s_{\alpha}, s_{\beta}\right)
$$

(where $x_{\alpha}(t)$ denotes the position of the wave $s_{\alpha}$ belonging to the $k_{\alpha}$-th characteristic family), thanks again to the analysis in $[7, \S 4]$ and [8] we deduce that, in the same setting of Lemma 1, the variation of $\mathcal{Q}$ corresponding to the 
incoming waves $s_{1}^{\prime}, \ldots, s_{N}^{\prime}, s_{1}^{\prime \prime}, \ldots, s_{N}^{\prime \prime}$, and to the outgoing waves $s_{1}, \ldots, s_{N}$ is bounded by

$$
\Delta \mathcal{Q} \leq-c_{0}\left[\sum_{\substack{1 \leq i, j \leq N \\ i>j}}\left|s_{i}^{\prime} s_{j}^{\prime \prime}\right|+\sum_{\substack{1 \leq i \leq N \\ s_{i}^{\prime} s_{i}^{\prime \prime}<0}} \mathcal{J}\left(s_{i}^{\prime}, s_{i}^{\prime \prime}\right)+\sum_{\substack{1 \leq i \leq N \\ s_{i}^{\prime} s_{i}^{\prime \prime}>0}} \mathcal{I}^{e}\left(s_{i}^{\prime}, s_{i}^{\prime \prime}\right)\right],
$$

for some constant $c_{0}>0$.

Relying on the estimates (3.2) and (3.26), (3.28) we are now ready to show that, given a Glimm approximate solution $u^{\epsilon}$, the interaction potential $Q$ in (3.25) is decreasing across the grid-times $i \varepsilon$, and that the variation of the total strength of waves $V$ in $u^{\epsilon}$ is controlled by $|\Delta Q|$.

Proposition 1. In the same setting of Lemma 1, there exist constants $\chi_{2}, c_{1}>$ 0 , and $c>0$ in (3.25) such that, setting $\Delta V \doteq V^{+}-V^{-}, \Delta Q \doteq Q^{+}-Q_{1}^{-}$, where $V^{-}, Q^{-}$and $V^{+}, Q^{+}$denote the values of $V, Q$ related, respectively, to the incoming waves $s_{1}^{\prime}, \ldots, s_{N}^{\prime}, s_{1}^{\prime \prime}, \ldots, s_{N}^{\prime \prime}$, and to the outgoing waves $s_{1}, \ldots, s_{N}$, and assuming $V^{-} \leq \chi_{2}$, there hold

$$
\begin{aligned}
& \Delta V \leq-c_{1} \cdot \sum_{i=1}^{N} \mathcal{C}\left(s_{i}^{\prime}, s_{i}^{\prime \prime}\right)+\mathcal{O}(1) \cdot\left[\sum_{\substack{1 \leq i, j \leq N \\
i>j}}\left|s_{i}^{\prime} s_{j}^{\prime \prime}\right|+\sum_{\substack{1 \leq i \leq N \\
s_{i}^{\prime} s_{i}^{\prime \prime}>0}} \mathcal{I}^{e}\left(s_{i}^{\prime}, s_{i}^{\prime \prime}\right)\right] \\
& \Delta Q \leq-c_{1} \cdot\left[\sum_{\substack{1 \leq i, j \leq N \\
i>j}}\left|s_{i}^{\prime} s_{j}^{\prime \prime}\right|+\sum_{\substack{1 \leq i \leq N \\
s_{i}^{\prime} s_{i}^{\prime \prime}>0}} \frac{\mathcal{I}^{e}\left(s_{i}^{\prime}, s_{i}^{\prime \prime}\right)}{\left|s_{i}^{\prime}\right|+\left|s_{i}^{\prime \prime}\right|}\right]+\mathcal{O}(1) \cdot V^{-} \cdot \sum_{i=1}^{N} \mathcal{C}\left(s_{i}^{\prime}, s_{i}^{\prime \prime}\right) .
\end{aligned}
$$

Proof. Observing that by (2.19) one has

$$
\sum_{\substack{1 \leq i \leq N \\ s_{i}^{\prime} s_{i}^{\prime \prime}<0}} \mathcal{J}\left(s_{i}^{\prime}, s_{i}^{\prime \prime}\right)=\mathcal{O}(1) \cdot \sum_{\substack{1 \leq i \leq N \\ s_{i}^{\prime} s_{i}^{\prime \prime}<0}}\left|s_{i}^{\prime} s_{i}^{\prime \prime}\right|=\mathcal{O}(1) \cdot V^{-} \cdot \sum_{i=1}^{N} \mathcal{C}\left(s_{i}^{\prime}, s_{i}^{\prime \prime}\right)
$$

we deduce that (3.29) is an immediate consequence of (3.2) and (3.26), (3.31), provided that $V^{-}$is sufficiently small. Thus, we will focus our attention on the estimate (3.30). For sake of simplicity, we shall consider only the case in which the two adjacent Riemann problems $\left(u^{L}, u^{M}\right),\left(u^{M}, u^{R}\right)$ are solved by a single wave, say $s^{\prime}$ and $s^{\prime \prime}, s^{\prime}$ on the left of $s^{\prime \prime}$, so that we have $V^{-}=\left|s^{\prime}\right|+\left|s^{\prime \prime}\right|$. We distinguish three cases, depending on the characteristic families of $s^{\prime}$ and $s^{\prime \prime}$ and on their sign sizes. 
1. $s^{\prime}$ and $s^{\prime \prime}$ are waves of the $k^{\prime}$ and $k^{\prime \prime}<k^{\prime}$ characteristic families.

Relying on (3.2), (3.24), we find that the variation of $Q$ is bounded by

$$
\begin{aligned}
\Delta Q= & {\left[\frac{\mathcal{I}\left(s_{k^{\prime}}, s_{k^{\prime}}\right)}{2\left|s_{k^{\prime}}\right|}-\frac{\mathcal{I}\left(s^{\prime}, s^{\prime}\right)}{2\left|s^{\prime}\right|}\right]+\left[\frac{\mathcal{I}\left(s_{k^{\prime \prime}}, s_{k^{\prime \prime}}\right)}{2\left|s_{k^{\prime \prime}}\right|}-\frac{\mathcal{I}\left(s^{\prime \prime}, s^{\prime \prime}\right)}{2\left|s^{\prime \prime}\right|}\right]-c\left|s^{\prime} s^{\prime \prime}\right| } \\
\leq & \frac{\left|\mathcal{I}\left(s_{k^{\prime}}, s_{k^{\prime}}\right)-\mathcal{I}\left(s^{\prime}, s^{\prime}\right)\right|}{2\left|s^{\prime}\right|}+\frac{\left|\mathcal{I}\left(s_{k^{\prime \prime}}, s_{k^{\prime \prime}}\right)-\mathcal{I}\left(s^{\prime \prime}, s^{\prime \prime}\right)\right|}{2\left|s^{\prime \prime}\right|}+ \\
+ & \mathcal{O}(1) \cdot\left[\frac{\mathcal{I}\left(s_{k^{\prime}}, s_{k^{\prime}}\right)}{\left|s^{\prime}\right|^{2}}+\frac{\mathcal{I}\left(s_{k^{\prime \prime}}, s_{k^{\prime \prime}}\right)}{\left.\left|s^{\prime \prime}\right|\right|^{2}}\right] \cdot\left[\left|s^{\prime}-s_{k^{\prime}}\right|+\left|s^{\prime \prime}-s_{k^{\prime \prime}}\right|\right]-c\left|s^{\prime} s^{\prime \prime}\right| \\
\leq & \frac{\left|\mathcal{I}\left(s_{k^{\prime}}, s_{k^{\prime}}\right)-\mathcal{I}\left(s^{\prime}, s^{\prime}\right)\right|}{2\left|s^{\prime}\right|}+\frac{\left|\mathcal{I}\left(s_{k^{\prime \prime}}, s_{k^{\prime \prime}}\right)-\mathcal{I}\left(s^{\prime \prime}, s^{\prime \prime}\right)\right|}{2\left|s^{\prime \prime}\right|}+ \\
& \quad+\mathcal{O}(1) \cdot\left[\left|s^{\prime}\right|+\left|s^{\prime \prime}\right|\right] \cdot\left|s^{\prime} s^{\prime \prime}\right|-c\left|s^{\prime} s^{\prime \prime}\right| .
\end{aligned}
$$

Set $u^{\sharp} \doteq T_{k^{\prime \prime}}\left[u^{L}\right]\left(s_{k^{\prime \prime}}\right)$, call $\sigma_{k^{\prime}} \doteq \sigma_{k^{\prime}}\left[u^{L}\right]\left(s_{k^{\prime}}, \cdot\right), \sigma^{\prime} \doteq \sigma_{k^{\prime}}\left[u^{\sharp}\right]\left(s^{\prime}, \cdot\right)$ the wavespeed maps of $s_{k^{\prime}}, s^{\prime}$, and denote by $\left\|\sigma_{k^{\prime}}-\sigma^{\prime}\right\|_{\infty}$ the $\mathbb{L}^{\infty}$ norm relative to the maximal common interval of definition of $\sigma_{k^{\prime}}, \sigma^{\prime}$. Then, by the Lipschitz continuity of $(u, s) \mapsto \sigma_{k}\left[u^{L}\right](s, \cdot)$, and because of $(3.2)$, we derive

$$
\begin{aligned}
\left|\mathcal{I}\left(s_{k^{\prime}}, s_{k^{\prime}}\right)-\mathcal{I}\left(s^{\prime}, s^{\prime}\right)\right| & =\mathcal{O}(1) \cdot\left[\left|s^{\prime}\right|^{2}\left\|\sigma_{k^{\prime}}-\sigma^{\prime}\right\|_{\infty}+\left(\left|s^{\prime}\right|+\left|s_{k^{\prime}}\right|\right) \cdot\left|s^{\prime}-s_{k^{\prime}}\right|\right] \\
& =\mathcal{O}(1) \cdot\left|s^{\prime}\right|^{2}\left|s^{\prime \prime}\right| .
\end{aligned}
$$

With the same arguments, and adopting the same notations, we get

$$
\begin{aligned}
\left|\mathcal{I}\left(s_{k^{\prime \prime}}, s_{k^{\prime \prime}}\right)-\mathcal{I}\left(s^{\prime \prime}, s^{\prime \prime}\right)\right| & =\mathcal{O}(1) \cdot\left[\left|s^{\prime \prime}\right|^{2}\left\|\sigma_{k^{\prime \prime}}-\sigma^{\prime \prime}\right\|_{\infty}+\left(\left|s^{\prime \prime}\right|+\left|s_{k^{\prime \prime}}\right|\right) \cdot\left|s^{\prime \prime}-s_{k^{\prime \prime}}\right|\right] \\
& =\mathcal{O}(1) \cdot\left|s^{\prime}\right|\left|s^{\prime \prime}\right|^{2} .
\end{aligned}
$$

Hence, (3.32)-(3.34) together yield

$$
\Delta Q \leq \mathcal{O}(1) \cdot\left|s^{\prime} s^{\prime \prime}\right|-c\left|s^{\prime} s^{\prime \prime}\right|
$$

which proves (3.30) provided that $c>0$ in (3.25) is chosen sufficiently large.

2. $s^{\prime}$ and $s^{\prime \prime}$ are both $k$-waves and $s^{\prime} s^{\prime \prime}<0$.

To fix the ideas, assume that $s^{\prime}>0$. We shall consider three cases.

(a) $\left|s^{\prime \prime}\right| \leq\left|s^{\prime}+s^{\prime \prime}\right|$.

In this case, one has $-s^{\prime}<s^{\prime \prime}<0, s^{\prime}+s^{\prime \prime}>0$, and $\mathcal{C}\left(s^{\prime}, s^{\prime \prime}\right)=\left|s^{\prime \prime}\right|$.

Using (3.2), (3.24), and relying on (2.19), we find that the variation of $Q$ 
is bounded by

$$
\begin{aligned}
\Delta Q= & \frac{\mathcal{I}\left(s_{k}, s_{k}\right)}{2\left|s_{k}\right|}-\left[\frac{\mathcal{I}\left(s^{\prime}, s^{\prime}\right)}{2\left|s^{\prime}\right|}+\frac{\mathcal{I}\left(s^{\prime \prime}, s^{\prime \prime}\right)}{2\left|s^{\prime \prime}\right|}+\frac{2 \mathcal{I}\left(s^{\prime}, s^{\prime \prime}\right)}{\left|s^{\prime}\right|+\left|s^{\prime \prime}\right|}\right] \\
\leq & \frac{\mathcal{I}\left(s_{k}, s_{k}\right)}{2\left|s^{\prime}+s^{\prime \prime}\right|}-\left[\frac{\mathcal{I}\left(s^{\prime}, s^{\prime}\right)}{2\left|s^{\prime}\right|}+\frac{\mathcal{I}\left(s^{\prime \prime}, s^{\prime \prime}\right)}{2\left|s^{\prime}+s^{\prime \prime}\right|}+\frac{2 \mathcal{I}\left(s^{\prime}, s^{\prime \prime}\right)}{\left|s^{\prime}\right|+\left|s^{\prime \prime}\right|}\right]+ \\
& \quad+\mathcal{O}(1) \cdot \frac{\mathcal{I}\left(s_{k}, s_{k}\right)}{\left|s^{\prime}+s^{\prime \prime}\right|^{2}} \cdot\left|s_{k}-\left(s^{\prime}+s^{\prime \prime}\right)\right| \\
\leq & \frac{\mathcal{I}\left(s_{k}, s_{k}\right)}{2\left|s^{\prime}+s^{\prime \prime}\right|}-\left[\frac{\mathcal{I}\left(s^{\prime}, s^{\prime}\right)}{2\left|s^{\prime}\right|}+\frac{\mathcal{I}\left(s^{\prime \prime}, s^{\prime \prime}\right)}{2\left|s^{\prime}+s^{\prime \prime}\right|}+\frac{2 \mathcal{I}\left(s^{\prime}, s^{\prime \prime}\right)}{\left|s^{\prime}\right|+\left|s^{\prime \prime}\right|}\right]+ \\
& \quad+\mathcal{O}(1) \cdot\left|s^{\prime}+s^{\prime \prime}\right| \cdot \mathcal{J}\left(s^{\prime}, s^{\prime \prime}\right) \\
\leq & \frac{\mathcal{I}\left(s_{k}, s_{k}\right)}{2\left|s^{\prime}+s^{\prime \prime}\right|}-\left[\frac{\mathcal{I}\left(s^{\prime}, s^{\prime}\right)}{2\left|s^{\prime}\right|}+\frac{\mathcal{I}\left(s^{\prime \prime}, s^{\prime \prime}\right)}{2\left|s^{\prime}+s^{\prime \prime}\right|}+\frac{2 \mathcal{I}\left(s^{\prime}, s^{\prime \prime}\right)}{\left|s^{\prime}\right|+\left|s^{\prime \prime}\right|}\right]+\mathcal{O}(1) \cdot\left|s^{\prime} s^{\prime \prime}\right| .
\end{aligned}
$$

On the other hand, since $\left|s^{\prime}\right|=\left|s^{\prime}+s^{\prime \prime}\right|+\left|s^{\prime \prime}\right| \leq 2\left|s^{\prime}+s^{\prime \prime}\right|$, applying (3.23), (3.24) we derive

$$
\begin{aligned}
\frac{\mathcal{I}\left(s^{\prime}, s^{\prime}\right)}{2\left|s^{\prime}\right|} & =\frac{\mathcal{I}\left(s^{\prime}, s^{\prime}\right)}{2\left|s^{\prime}+s^{\prime \prime}\right|}+\mathcal{O}(1) \cdot \frac{\mathcal{I}\left(s^{\prime}, s^{\prime}\right)}{\left|s^{\prime}+s^{\prime \prime}\right|^{2}} \cdot\left|s^{\prime \prime}\right| \\
& =\frac{\mathcal{I}\left(s^{\prime}, s^{\prime}\right)}{2\left|s^{\prime}+s^{\prime \prime}\right|}+\mathcal{O}(1) \cdot\left|s^{\prime} s^{\prime \prime}\right|, \\
\frac{\mathcal{I}\left(s^{\prime}, s^{\prime \prime}\right)}{\left|s^{\prime}\right|+\left|s^{\prime \prime}\right|} & =\frac{\mathcal{I}\left(s^{\prime}, s^{\prime \prime}\right)}{2\left|s^{\prime}+s^{\prime \prime}\right|}+\mathcal{O}(1) \cdot \frac{\mathcal{I}\left(s^{\prime}, s^{\prime \prime}\right)}{\left|s^{\prime}+s^{\prime \prime}\right|^{2}} \cdot\left|s^{\prime \prime}\right| \\
& =\frac{\mathcal{I}\left(s^{\prime}, s^{\prime \prime}\right)}{2\left|s^{\prime}+s^{\prime \prime}\right|}+\mathcal{O}(1) \cdot\left|s^{\prime} s^{\prime \prime}\right|,
\end{aligned}
$$

which, together with (3.36), yields

$$
\Delta Q \leq \frac{\mathcal{I}\left(s_{k}, s_{k}\right)-\left[\mathcal{I}\left(s^{\prime}, s^{\prime}\right)+\mathcal{I}\left(s^{\prime \prime}, s^{\prime \prime}\right)+2 \mathcal{I}\left(s^{\prime}, s^{\prime \prime}\right)\right]}{2\left|s^{\prime}+s^{\prime \prime}\right|}+\mathcal{O}(1) \cdot\left|s^{\prime} s^{\prime \prime}\right|
$$

Notice now that, recalling definition (3.27), and thanks to (3.28) one has

$$
\begin{gathered}
\mathcal{I}\left(s_{k}, s_{k}\right)-\left[\mathcal{I}\left(s^{\prime}, s^{\prime}\right)+\mathcal{I}\left(s^{\prime \prime}, s^{\prime \prime}\right)+2 \mathcal{I}\left(s^{\prime}, s^{\prime \prime}\right)\right]= \\
=4 \Delta \mathcal{Q}<0 .
\end{gathered}
$$

Hence, from (3.39)-(3.40) we recover (3.30) since $\left|s^{\prime} s^{\prime \prime}\right| \leq V^{-} \cdot \mathcal{C}\left(s^{\prime}, s^{\prime \prime}\right)$. 
(b) $\left|s^{\prime}\right| \leq\left|s^{\prime}+s^{\prime \prime}\right|<\left|s^{\prime \prime}\right|$.

In this case, one has $s^{\prime \prime}<s^{\prime}+s^{\prime \prime} \leq-s^{\prime}<0$, and $\mathcal{C}\left(s^{\prime}, s^{\prime \prime}\right)=\left|s^{\prime}\right|$. Using (3.2), (3.23), (3.24), relying on (2.19), and observing that $\left|s^{\prime \prime}\right|=$ $\left|s^{\prime}+s^{\prime \prime}\right|+\left|s^{\prime}\right| \leq 2\left|s^{\prime}+s^{\prime \prime}\right|$, with similar computations as in (3.36)-(3.38) we find that

$$
\begin{aligned}
\Delta Q= & \frac{\mathcal{I}\left(s_{k}, s_{k}\right)}{2\left|s_{k}\right|}-\left[\frac{\mathcal{I}\left(s^{\prime}, s^{\prime}\right)}{2\left|s^{\prime}\right|}+\frac{\mathcal{I}\left(s^{\prime \prime}, s^{\prime \prime}\right)}{2\left|s^{\prime \prime}\right|}+\frac{2 \mathcal{I}\left(s^{\prime}, s^{\prime \prime}\right)}{\left|s^{\prime}\right|+\left|s^{\prime \prime}\right|}\right] \\
\leq & \frac{\mathcal{I}\left(s_{k}, s_{k}\right)}{2\left|s^{\prime}+s^{\prime \prime}\right|}-\left[\frac{\mathcal{I}\left(s^{\prime}, s^{\prime}\right)}{2\left|s^{\prime}+s^{\prime \prime}\right|}+\frac{\mathcal{I}\left(s^{\prime \prime}, s^{\prime \prime}\right)}{2\left|s^{\prime \prime}\right|}+\frac{2 \mathcal{I}\left(s^{\prime}, s^{\prime \prime}\right)}{\left|s^{\prime}\right|+\left|s^{\prime \prime}\right|}\right]+ \\
& \quad+\mathcal{O}(1) \cdot \frac{\mathcal{I}\left(s_{k}, s_{k}\right)}{\left|s^{\prime}+s^{\prime \prime}\right|^{2}} \cdot\left|s_{k}-\left(s^{\prime}+s^{\prime \prime}\right)\right| \\
\leq & \frac{\mathcal{I}\left(s_{k}, s_{k}\right)}{2\left|s^{\prime}+s^{\prime \prime}\right|}-\left[\frac{\mathcal{I}\left(s^{\prime}, s^{\prime}\right)}{2\left|s^{\prime}+s^{\prime \prime}\right|}+\frac{\mathcal{I}\left(s^{\prime \prime}, s^{\prime \prime}\right)}{2\left|s^{\prime \prime}\right|}+\frac{2 \mathcal{I}\left(s^{\prime}, s^{\prime \prime}\right)}{\left|s^{\prime}\right|+\left|s^{\prime \prime}\right|}\right]+\mathcal{O}(1) \cdot\left|s^{\prime} s^{\prime \prime}\right|,
\end{aligned}
$$

and

$$
\begin{aligned}
\frac{\mathcal{I}\left(s^{\prime \prime}, s^{\prime \prime}\right)}{2\left|s^{\prime \prime}\right|} & =\frac{\mathcal{I}\left(s^{\prime \prime}, s^{\prime \prime}\right)}{2\left|s^{\prime}+s^{\prime \prime}\right|}+\mathcal{O}(1) \cdot \frac{\mathcal{I}\left(s^{\prime \prime}, s^{\prime \prime}\right)}{\left|s^{\prime}+s^{\prime \prime}\right|^{2}} \cdot\left|s^{\prime}\right| \\
& =\frac{\mathcal{I}\left(s^{\prime \prime}, s^{\prime \prime}\right)}{2\left|s^{\prime}+s^{\prime \prime}\right|}+\mathcal{O}(1) \cdot\left|s^{\prime} s^{\prime \prime}\right|, \\
\frac{\mathcal{I}\left(s^{\prime}, s^{\prime \prime}\right)}{\left|s^{\prime}\right|+\left|s^{\prime \prime}\right|} & =\frac{\mathcal{I}\left(s^{\prime}, s^{\prime \prime}\right)}{2\left|s^{\prime}+s^{\prime \prime}\right|}+\mathcal{O}(1) \cdot \frac{\mathcal{I}\left(s^{\prime}, s^{\prime \prime}\right)}{\left|s^{\prime}+s^{\prime \prime}\right|^{2}} \cdot\left|s^{\prime}\right| \\
& =\frac{\mathcal{I}\left(s^{\prime}, s^{\prime \prime}\right)}{2\left|s^{\prime}+s^{\prime \prime}\right|}+\mathcal{O}(1) \cdot\left|s^{\prime} s^{\prime \prime}\right| .
\end{aligned}
$$

Hence, relying on (3.28) and (3.41)-(3.43), we derive (3.39)-(3.40), which imply (3.30) since $\left|s^{\prime} s^{\prime \prime}\right| \leq V^{-} \cdot \mathcal{C}\left(s^{\prime}, s^{\prime \prime}\right)$.

(c) $\left|s^{\prime}+s^{\prime \prime}\right| \leq \min \left\{\left|s^{\prime}\right|,\left|s^{\prime \prime}\right|\right\}$.

In this case, using (3.2), (3.24), we derive

$$
\begin{aligned}
\Delta Q & \leq \frac{\mathcal{I}\left(s_{k}, s_{k}\right)}{2\left|s_{k}\right|}=\mathcal{O}(1) \cdot\left|s_{k}\right|^{2} \\
& =\mathcal{O}(1) \cdot\left[\left|s^{\prime}+s^{\prime \prime}\right|^{2}+\left|s^{\prime} s^{\prime \prime}\right|\right] \\
& =\mathcal{O}(1) \cdot\left|s^{\prime} s^{\prime \prime}\right|,
\end{aligned}
$$

which proves (3.30) since we have $\left|s^{\prime} s^{\prime \prime}\right| \leq V^{-} \cdot \mathcal{C}\left(s^{\prime}, s^{\prime \prime}\right)$. 
3. $s^{\prime}$ and $s^{\prime \prime}$ are both $k$-waves and $s^{\prime} s^{\prime \prime}>0$.

Using (3.2), (3.24), and relying on (3.26), (3.28), with similar computations as above we find

$$
\begin{aligned}
\Delta Q= & \frac{\mathcal{I}\left(s_{k}, s_{k}\right)}{2\left|s_{k}\right|}-\left[\frac{\mathcal{I}\left(s^{\prime}, s^{\prime}\right)}{2\left|s^{\prime}\right|}+\frac{\mathcal{I}\left(s^{\prime \prime}, s^{\prime \prime}\right)}{2\left|s^{\prime \prime}\right|}+\frac{2 \mathcal{I}\left(s^{\prime}, s^{\prime \prime}\right)}{\left|s^{\prime}\right|+\left|s^{\prime \prime}\right|}\right] \\
\leq & \frac{\mathcal{I}\left(s_{k}, s_{k}\right)-\left[\mathcal{I}\left(s^{\prime}, s^{\prime}\right)+\mathcal{I}\left(s^{\prime \prime}, s^{\prime \prime}\right)+2 \mathcal{I}\left(s^{\prime}, s^{\prime \prime}\right)\right]}{2\left(\left|s^{\prime}\right|+\left|s^{\prime \prime}\right|\right)}+ \\
& \quad+\mathcal{O}(1) \cdot \frac{\mathcal{I}\left(s_{k}, s_{k}\right)}{\left(\left|s^{\prime}\right|+\left|s^{\prime \prime}\right|\right)^{2}} \cdot\left|s_{k}-\left(s^{\prime}+s^{\prime \prime}\right)\right| \\
\leq & \frac{\mathcal{I}\left(s_{k}, s_{k}\right)-\left[\mathcal{I}\left(s^{\prime}, s^{\prime}\right)+\mathcal{I}\left(s^{\prime \prime}, s^{\prime \prime}\right)+2 \mathcal{I}\left(s^{\prime}, s^{\prime \prime}\right)\right]}{2\left(\left|s^{\prime}\right|+\left|s^{\prime \prime}\right|\right)}+\mathcal{O}(1) \cdot \mathcal{J}\left(s^{\prime}, s^{\prime \prime}\right) \\
\leq & {\left[-\frac{c_{0}}{2}+\mathcal{O}(1) \cdot\left(\left|s^{\prime}\right|+\left|s^{\prime \prime}\right|\right)\right] \cdot \frac{\mathcal{I}^{e}\left(s^{\prime}, s^{\prime \prime}\right)}{\left|s^{\prime}\right|+\left|s^{\prime \prime}\right|}, }
\end{aligned}
$$

which proves (3.30) assuming $V^{-}$sufficiently small. This completes the proof of the proposition.

An immediate consequence of Proposition 1 is the following

Corollary 1. For every compact set $K \subset \Omega$, there exist constants $\chi_{3}, c_{2}, C>0$ such that the following holds. Let $u^{\varepsilon}=u^{\varepsilon}(t, x)$ be a Glimm approximate solution of $(1.4),(1.6)$, defined on the strip $[0, i \varepsilon] \times \mathbb{R}$, suppose that no splitting of a rarefaction component of a wave in $u^{\varepsilon}(i \varepsilon-, \cdot)$ is determined by the sampling $\theta_{i}$, and assume that Tot.Var. $\left\{u^{\varepsilon}(i \varepsilon-, \cdot)\right\}<\chi_{3}, \lim _{x \rightarrow-\infty} u^{\varepsilon}(i \varepsilon-, x) \in K$. Then, letting $V^{-} \doteq V(i \varepsilon-), Q^{-} \doteq Q(i \varepsilon-)$ and $V^{+} \doteq V(i \varepsilon+), Q^{+} \doteq Q(i \varepsilon+)$ denote the values of $V, Q$ related to $u^{\varepsilon}(i \varepsilon-, \cdot)$ and $u^{\varepsilon}(i \varepsilon+, \cdot)$, respectively, and setting $\Delta V(i \varepsilon) \doteq V^{+}-V^{-}, \Delta Q(i \varepsilon) \doteq Q^{+}-Q^{-}$, there hold

$$
[\Delta V+C \cdot \Delta Q](i \varepsilon) \leq-c_{2} \cdot\left[\sum_{\substack{k_{\alpha}<k_{\beta} \\ x_{\alpha}>x_{\beta}}}\left|s_{\alpha} s_{\beta}\right|+\sum_{k_{\alpha}=k_{\beta}} \mathcal{C}\left(s_{\alpha}, s_{\beta}\right)+\sum_{\substack{k_{\alpha}=k_{\beta} \\ s_{\alpha} \cdot s_{\beta}>0}} \frac{\mathcal{I}^{e}\left(s_{\alpha}, s_{\beta}\right)}{\left|s_{\alpha}\right|+\left|s_{\beta}\right|}\right] \text {, }
$$

where $s_{\alpha}$ denotes a wave in $u^{\varepsilon}(i \varepsilon-, \cdot)$ of the $k_{\alpha}$-th family located in $x_{\alpha}$.

Remark 6. Consider two adjacent Riemann problems $\left(w^{j-1}, w^{j}\right),\left(w^{j}, w^{j+1}\right)$, with $w^{j-1} \doteq u^{\varepsilon}(i \varepsilon+,(j-1) \varepsilon), w^{j} \doteq u^{\varepsilon}(i \varepsilon+, j \varepsilon), w^{j+1} \doteq u^{\varepsilon}(i \varepsilon+,(j+1) \varepsilon)$, in a Glimm approximate solution $u^{\varepsilon}$. Assume that the fastest wave $s_{\alpha}$ of $\left(w^{j-1}, w^{j}\right)$ belongs to the same family of the slowest wave $s_{\beta}$ of $\left(w^{j}, w^{j+1}\right)$, and suppose that they are not interacting, i.e. that $\mathcal{J}\left(s_{\alpha}, s_{\beta}\right)=\mathcal{I}^{e}\left(s_{\alpha}, s_{\beta}\right)=0$. Then, it will be convenient to treat $s_{\alpha}$ and $s_{\beta}$ as a single wave when considering their 
contribution to the cubic part of the potential interaction (3.25). Thanks to this choice the functional $Q$ remains unchanged when it occurs the splitting of a wave determined by the sampling procedure. On the other hand, in this way one has to pay attention to the fact that the entire effect on the functional $Q$ of an interaction of one of the two waves $s_{\alpha}, s_{\beta}$ with another wave, say $s_{\gamma}$, can be computed only when $s_{\gamma}$ has concluded the interaction with both waves $s_{\alpha}, s_{\beta}$.

By Corollary 1, and in view of the Remark 6, it follows that if $V(t), Q(t)$ denote the total strength of waves (3.18) and the interaction potential (3.25) of an approximate solution $u^{\epsilon}(t)$ constructed by the Glimm scheme, the functional

$$
t \mapsto \Upsilon(t) \doteq V(t)+C \cdot Q(t)
$$

is non increasing in time provided that the initial strength $V(0)$ is sufficiently small. Moreover, for any given $0 \leq m<n$, the total amount of wave interaction and cancellation taking place in the time interval $[m \varepsilon, n \varepsilon]$ is bounded by $\mathcal{O}(1) \cdot\left|\Upsilon^{m, n}\right|$, where

$$
\Delta \Upsilon^{m, n} \doteq \Upsilon(n \varepsilon+)-\Upsilon(m \varepsilon+)
$$

denotes the variation of $\Upsilon$ on $[m \varepsilon, n \varepsilon]$.

\section{Wave tracing for general quasilinear systems}

We will show now how to implement a wave tracing algorithm for a general quasilinear system (1.1) so that the change in strength and the product of strength times the variation in speeds of the primary waves be bounded by the variation of the Glimm functional in (3.47). Namely, recalling the Definition 2 of a wave partition, we have the following result analogous to [5, Proposition 2].

Proposition 2. Given a Glimm approximate solution and any fixed $0 \leq m<$ $n$, there exists a partition of elementary wave sizes and speeds $\left\{y_{k}^{h}(i, j), \lambda_{k}^{h}(i, j)\right\}$, $k=1, \ldots, N, i=m, m+1, \ldots, n, j \in \mathbb{Z}$, so that the following hold.

1. For every $i, j, k,\left\{y_{k}^{h}(i, j)\right\}_{0<h \leq \ell_{k}(i, j)}$ is a partition of the wave of the $k$-th family issuing from $(i \varepsilon, j \varepsilon)$, and $\left\{\lambda_{k}^{h}(i, j)\right\}_{0<h \leq \ell_{k}(i, j)}$ are the corresponding speeds, according with Definition 2.

2. For every $i, j, k,\left\{y_{k}^{h}(i, j), \lambda_{k}^{h}(i, j)\right\}_{0<h \leq \ell_{k}(i, j)}$ is a disjoint union of the two sets

$$
\left\{\widetilde{y}_{k}^{h}(i, j), \widetilde{\lambda}_{k}^{h}(i, j)\right\}, \quad\left\{\widetilde{\widetilde{y}}_{k}^{h}(i, j), \widetilde{\widetilde{\lambda}}_{k}^{h}(i, j)\right\}
$$

with the following properties:

(a)

$$
\sum_{j, k, h}\left\|\widetilde{\widetilde{y}}_{k}^{h}(i, j)\right\|=\mathcal{O}(1) \cdot\left|\Delta \Upsilon^{m, n}\right| \quad \forall m \leq i \leq n
$$


(b) for every fixed $i, k, h$, there is a one-to-one correspondence between $\left\{\widetilde{y}_{k}^{h}(m, j), \widetilde{\lambda}_{k}^{h}(m, j) ; j \in \mathbb{Z}\right\}$ and $\left\{\widetilde{y}_{k}^{h}(i, j), \widetilde{\lambda}_{k}^{h}(i, j) ; j \in \mathbb{Z}\right\}:$

$$
\left\{\widetilde{y}_{k}^{h}(m, j), \widetilde{\lambda}_{k}^{h}(m, j)\right\} \leftrightarrow\left\{\widetilde{y}_{k}^{h}\left(i, \ell_{(i, j, k, h)}\right), \widetilde{\lambda}_{k}^{h}\left(i, \ell_{(i, j, k, h)}\right)\right\}
$$

such that the sizes $\widetilde{s}_{k}^{h}$ and the speeds $\widetilde{\lambda}_{k}^{h}$ of the corresponding waves satisfy

$$
\begin{gathered}
\sum_{j, k, h}\left(\max _{m \leq i \leq n}\left|\widetilde{s}_{k}^{h}(m, j)-\widetilde{s}_{k}^{h}\left(i, \ell_{(i, j, k, h)}\right)\right|\right)=\mathcal{O}(1) \cdot\left|\Delta \Upsilon^{m, n}\right|, \\
\sum_{j, k, h}\left(\left|\widetilde{s}_{k}^{h}(m, j)\right| \cdot \max _{m \leq i \leq n}\left|\widetilde{\lambda}_{k}^{h}(m, j)-\widetilde{\lambda}_{k}^{h}\left(i, \ell_{(i, j, k, h)}\right)\right|\right)=\mathcal{O}(1) \cdot\left|\Delta \Upsilon^{m, n}\right|,
\end{gathered}
$$

where $\Delta \Upsilon^{m, n}$ is the variation (3.48) of the functional $\Upsilon$.

Proof. In order to produce a partition for an approximate solution $u^{\varepsilon}$ that fulfills properties 1-2, we shall proceed by induction on the time steps $i \varepsilon$, $m \leq i<n$. Then, assuming that such a partition is given for all times $m \varepsilon \leq t<i \varepsilon$, our goal is to show how to define a partition of the outgoing waves generated by the interactions that take place at $t=i \varepsilon$, preserving the properties 1-2. It will be sufficient to focus our attention on interactions between waves of the same family, since for interactions between waves of different families the change in strength and the product of strength times the variation in speeds is controlled by the variation of a quadratic interaction potential as the first term in (3.25), and hence it is standard the definition of a partition verifying 1.-2. for the outgoing waves generated by an interaction of this type (cfr. [25, Theorem 5.1]).

Thus, consider an interaction between two waves, say $s_{k}^{\prime}, s_{k}^{\prime \prime}$, issuing from two consecutive mesh points $((i-1) \varepsilon,(j-1) \varepsilon)$ and $((i-1) \varepsilon, j \varepsilon)$, belonging to a $k$-th characteristic family. We shall distinguish two cases.

1. $s_{k}^{\prime}$ and $s_{k}^{\prime \prime}$ have the same sign.

For the sake of simplicity, we assume that $s_{k}^{\prime}, s_{k}^{\prime \prime}>0$ and that the outgoing $k$-wave $s_{k}$ is a shock, the other cases being entirely similar. Hence, by Definition 4, and relying on (3.2), (3.26), we have

$$
\mathcal{I}\left(s_{k}^{\prime}, s_{k}^{\prime \prime}\right)=\mathcal{I}^{e}\left(s_{k}^{\prime}, s_{k}^{\prime \prime}\right)+\mathcal{O}(1) \cdot\left[\left|s^{\prime}\right|+\left|s^{\prime \prime}\right|\right] \cdot \mathcal{I}^{e}\left(s_{k}^{\prime}, s_{k}^{\prime \prime}\right) .
$$

Let

$$
\left\{y_{k}^{\prime h}, \lambda_{k}^{\prime h}\right\}_{0<h \leq \ell^{\prime}}, \quad\left\{y_{k}^{\prime \prime h}, \lambda_{k}^{\prime \prime h}\right\}_{0<h \leq \ell^{\prime \prime}}
$$

be the partitions of $s_{k}^{\prime}$ and $s_{k}^{\prime \prime}$ enjoying the properties 1-2 (on the interval $[m \varepsilon,(i-1) \varepsilon])$, with sizes

$$
\left\{s_{k}^{\prime h}\right\}_{0<h \leq \ell^{\prime}}, \quad\left\{s_{k}^{\prime \prime h}\right\}_{0<h \leq \ell^{\prime \prime}} .
$$


For every $p \neq k$-th wave $s_{p}$, we may choose a partition $\left\{y_{p}^{h}\right\}_{0<h \leq \ell_{p}}$ as in Definition 2, with corresponding speeds $\left\{\lambda_{p}^{h}\right\}_{0<h \leq \ell_{p}}$. Then, if we label all the subwaves $y_{p}^{h}$ as secondary waves $\widetilde{\widetilde{y}}_{p}^{h}$, the bound (4.1) (for $i, j, p$ ) is certainly satisfied thanks to the interaction estimates (3.2) and to Corollary 1 . Instead, for the $k$-th wave $s_{k}$, possibly considering a refinement of the partition of $s_{k}^{\prime \prime}$ (or of $s_{k}^{\prime}$ ) we may assume that either $s_{k}^{\prime}+s_{k}^{\prime \prime 1} \leq s_{k}$, or $s_{k}^{\prime 1} \leq s_{k}$ (in the case $s_{k}^{\prime} \geq s_{k}$ ), and let $\bar{\ell}^{\prime} \doteq \max \left\{h \leq \ell^{\prime}: \sum_{q=1}^{h} s_{k}^{\prime q} \leq s_{k}\right\}$, $\bar{\ell}^{\prime \prime} \doteq \max \left\{h \leq \ell^{\prime \prime}: s_{k}^{\prime}+\sum_{q=1}^{h} s_{k}^{\prime \prime q} \leq s_{k}\right\}$. Then, we define a partition of $s_{k}$ by means of its sizes, setting

$$
s_{k}^{h} \doteq \begin{cases}s_{k}^{\prime h} & \text { if } \quad h=1, \ldots, \bar{\ell}^{\prime}, \\ s_{k}^{\prime \prime h-\ell^{\prime}} & \text { if } \quad \bar{\ell}^{\prime}=\ell^{\prime} \quad \text { and } \quad h=\ell^{\prime}+1, \ldots, \ell^{\prime}+\bar{\ell}^{\prime \prime},\end{cases}
$$

(possibly refining the partitions (4.6) so to satisfy property 2 of Definition 2), and choosing a partition of $s_{k}-\left(s_{k}^{\prime}+s_{k}^{\prime \prime}\right)$ as in Definition 2 in the case $s_{k}>s_{k}^{\prime}+s_{k}^{\prime \prime}$. The subwaves $s_{k}^{h}$ in (4.8) inherit the same classification in primary and secondary waves of the corresponding subwaves $s_{k}^{\prime h}$ or $s_{k}^{\prime \prime h-\ell^{\prime}}$, while all the possible subwaves of $s_{k}-\left(s_{k}^{\prime}+s_{k}^{\prime \prime}\right)$ are labeled as secondary waves.

Clearly, the bound (4.1) is again satisfied because of the interaction estimates (3.2), and thanks to Corollary 1, while the one-to-one correspondence at (4.2) and the bound (4.3) are verified by construction and by the inductive assumption. Hence, in order to conclude the proof, it remains to establish only the estimate (4.4) on the wave speeds. To this end, notice that the Rankine-Hugoniot speed $\lambda_{k}$ of the outgoing $k$-wave $s_{k}$ coincides with the speeds $\lambda_{k}^{h}$ of all subwaves $s_{k}^{h}$ defined according with Definition 2, since for a shock wave the integrand function $\sigma(\cdot)$ in (2.14) results to be a constant (cfr. Remark 1). Moreover, by the choice of the speeds of a partition at (2.14), one has

$$
\lambda^{\prime h}=\frac{1}{s_{k}^{\prime h}} \int_{\tau_{k}^{\prime h-1}}^{\tau_{k}^{\prime h}} \sigma^{\prime}(\xi) d \xi, \quad \lambda^{\prime \prime h}=\frac{1}{s_{k}^{\prime \prime h}} \int_{\tau_{k}^{\prime \prime h-1}}^{\tau_{k}^{\prime \prime h}} \sigma^{\prime \prime}(\xi) d \xi,
$$

where $\tau_{k}^{\prime h} \doteq \sum_{p=1}^{h} s_{k}^{\prime p}, \tau_{k}^{\prime \prime h} \doteq \sum_{p=1}^{h} s_{k}^{\prime \prime p}$, and $\sigma^{\prime}(\cdot) \doteq \sigma_{k}\left[\omega_{k}^{\prime}\right]\left(s_{k}^{\prime}, \cdot\right), \sigma^{\prime \prime}(\cdot) \doteq$ $\sigma_{k}\left[\omega_{k}^{\prime \prime}\right]\left(s_{k}^{\prime \prime}, \cdot\right)$, denote the map in (2.9) defining the speed of the rarefaction and shock components of $s_{k}^{\prime}$ and $s_{k}^{\prime \prime}$, respectively $\left(\omega_{k}^{\prime}, \omega_{k}^{\prime}\right.$ being the left states of $\left.s_{k}^{\prime}, s_{k}^{\prime \prime}\right)$. Then, applying Lemma 1 , one obtains the following estimate on the wave speeds (in the same spirit of the ones provided by $[25$, Theorem 3.1]):

$$
\begin{aligned}
\lambda_{k} \cdot\left(s_{k}^{\prime}+s_{k}^{\prime \prime}\right) & =\int_{0}^{s_{k}^{\prime}+s_{k}^{\prime \prime}} \sigma(\xi) d \xi+\mathcal{O}(1) \cdot \mathcal{J}\left(s_{k}^{\prime}, s_{k}^{\prime \prime}\right) \\
& =\int_{0}^{s_{k}^{\prime}} \sigma^{\prime}(\xi) d \xi+\int_{0}^{s_{k}^{\prime \prime}} \sigma^{\prime \prime}(\xi) d \xi+\mathcal{O}(1) \cdot \mathcal{J}\left(s_{k}^{\prime}, s_{k}^{\prime \prime}\right),
\end{aligned}
$$


which, relying on (4.9), yields

$$
\lambda_{k} \cdot\left(s_{k}^{\prime}+s_{k}^{\prime \prime}\right)=\sum_{h=1}^{\ell^{\prime}} s_{k}^{\prime h} \lambda_{k}^{\prime h}+\sum_{h=1}^{\ell^{\prime \prime}} s_{k}^{\prime \prime h} \lambda_{k}^{\prime \prime h}+\mathcal{O}(1) \cdot \mathcal{J}\left(s_{k}^{\prime}, s_{k}^{\prime \prime}\right) .
$$

Thus, since by the monotonicity property of $\sigma^{\prime}(\cdot)$ and $\sigma^{\prime \prime}(\cdot)$, we have

$$
\lambda_{k}^{\prime \prime h}-\mathcal{O}(1) \cdot \mathcal{J}\left(s_{k}^{\prime}, s_{k}^{\prime \prime}\right) \leq \lambda_{k} \leq \lambda_{k}^{\prime h}+\mathcal{O}(1) \cdot \mathcal{J}\left(s_{k}^{\prime}, s_{k}^{\prime \prime}\right) \quad \forall h,
$$

using (4.11) we derive

$$
\begin{aligned}
\left|\lambda_{k}^{\prime h}-\lambda_{k}\right|= & \lambda_{k}^{\prime h}-\lambda_{k}+\mathcal{O}(1) \cdot \mathcal{J}\left(s_{k}^{\prime}, s_{k}^{\prime \prime}\right) \\
= & \frac{1}{s_{k}^{\prime}+s_{k}^{\prime \prime}} \cdot\left[\sum_{p=1, \ldots, \ell^{\prime}} s_{k}^{\prime p}\left(\lambda_{k}^{\prime h}-\lambda_{k}^{\prime p}\right)+\sum_{p=1}^{\ell^{\prime \prime}} s_{k}^{\prime \prime p}\left(\lambda_{k}^{\prime h}-\lambda_{k}^{\prime \prime p}\right)\right]+ \\
& +\mathcal{O}(1) \cdot \frac{\mathcal{J}\left(s_{k}^{\prime}, s_{k}^{\prime \prime}\right)}{s_{k}^{\prime}+s_{k}^{\prime \prime}}
\end{aligned}
$$

which, in turn, yields

$$
\begin{aligned}
\sum_{h=1}^{\ell^{\prime}} s_{k}^{\prime h}\left|\lambda_{k}^{\prime h}-\lambda_{k}\right| & =\frac{1}{s_{k}^{\prime}+s_{k}^{\prime \prime}} \cdot\left[\sum_{h=1}^{\ell^{\prime}} \sum_{p=1}^{\ell^{\prime}} s_{k}^{\prime h} s_{k}^{\prime p}\left(\lambda_{k}^{\prime h}-\lambda_{k}^{\prime p}\right)+\right. \\
& \left.+\sum_{h=1}^{\ell^{\prime}} \sum_{p=1}^{\ell^{\prime \prime}} s_{k}^{\prime h} s_{k}^{\prime \prime p}\left(\lambda_{k}^{\prime h}-\lambda_{k}^{\prime \prime p}\right)\right]+\mathcal{O}(1) \cdot \mathcal{J}\left(s_{k}^{\prime}, s_{k}^{\prime \prime}\right) \cdot \frac{s_{k}^{\prime}}{s_{k}^{\prime}+s_{k}^{\prime \prime}}
\end{aligned}
$$

Notice that the terms of the first double sum on the right hand side of (4.12) are antisimmetric in $(h, p)$, and hence the first summand vanishes. Moreover, recalling (3.20), (4.9), and thanks to (4.5), we have

$$
\begin{aligned}
\sum_{h=1}^{\ell^{\prime}} & \sum_{p=1}^{\ell^{\prime \prime}} s_{k}^{\prime h} s_{k}^{\prime \prime p}\left(\lambda_{k}^{\prime h}-\lambda_{k}^{\prime \prime p}\right)= \\
& =\sum_{p=1}^{\ell^{\prime \prime}} s_{k}^{\prime \prime p} \sum_{h=1}^{\ell^{\prime}} \int_{\tau_{k}^{\prime h-1}}^{\tau_{k}^{\prime h}} \sigma^{\prime}(\xi) d \xi-\sum_{h=1}^{\ell^{\prime}} s_{k}^{\prime h} \sum_{p=1}^{\ell^{\prime \prime}} \int_{\tau_{k}^{\prime \prime h-1}}^{\tau_{k}^{\prime \prime h-1}} \sigma^{\prime \prime}(\eta) d \eta \\
& =s_{k}^{\prime \prime} \int_{0}^{s_{k}^{\prime}} \sigma^{\prime}(\xi) d \xi-s_{k}^{\prime} \int_{0}^{s_{k}^{\prime \prime}} \sigma^{\prime \prime}(\eta) d \eta \\
& =\int_{0}^{s_{k}^{\prime}} \int_{0}^{s_{k}^{\prime \prime}}\left[\sigma^{\prime}(\xi)-\sigma^{\prime \prime}(\eta)\right] d \eta d \xi \\
& =\mathcal{I}^{e}\left(s_{k}^{\prime}, s_{k}^{\prime \prime}\right)+\mathcal{O}(1) \cdot\left[\left|s_{k}^{\prime}\right|+\left|s_{k}^{\prime \prime}\right|\right] \cdot \mathcal{I}^{e}\left(s_{k}^{\prime}, s_{k}^{\prime \prime}\right) .
\end{aligned}
$$


Thus, from (4.12)-(4.13), and applying (3.46), we derive

$$
\begin{aligned}
\sum_{h=1}^{\ell^{\prime}} s_{k}^{\prime h}\left|\lambda_{k}^{\prime h}-\lambda_{k}\right| & =\frac{\mathcal{I}^{e}\left(s_{k}^{\prime}, s_{k}^{\prime \prime}\right)}{\left|s_{k}^{\prime}\right|+\left|s_{k}^{\prime \prime}\right|}+\mathcal{O}(1) \cdot \mathcal{I}^{e}\left(s_{k}^{\prime}, s_{k}^{\prime \prime}\right) \\
& =\mathcal{O}(1) \cdot|\Delta \Upsilon(i \varepsilon)|,
\end{aligned}
$$

where $\Delta \Upsilon$ is the variation of the functional $\Upsilon$ in (3.47). An entirely similar estimate can be obtained for the components of the partition of $s_{k}^{\prime \prime}$, so that there holds

$$
\sum_{h=1}^{\ell^{\prime \prime}} s_{k}^{\prime \prime h}\left|\lambda_{k}^{\prime \prime h}-\lambda_{k}\right|=\mathcal{O}(1) \cdot|\Delta \Upsilon(i \varepsilon)| .
$$

Therefore, relying on the inductive assumption, from (4.14)-(4.15) we recover the desired estimate (4.4) on the time interval $[m \varepsilon, i \varepsilon]$.

2. $s_{k}^{\prime}$ and $s_{k}^{\prime \prime}$ have opposite sign.

To fix the ideas, assume that $s_{k}^{\prime}>-s_{k}^{\prime \prime}>0$, the other cases being entirely similar. Adopting the above notations, we may define a partition $\left\{y_{p}^{h}\right\}_{0<h \leq \ell_{p}}$ of the outgoing wave $s_{p}(p=1, \ldots, N)$ of the $p$-th family issuing from $(i \varepsilon, j \varepsilon)$ (with corresponding speeds $\left\{\lambda_{p}^{h}\right\}_{0<h \leq \ell_{p}}$ ) as in the previous case of interacting waves with the same sign. In particular, possibly considering a refinement of the partition of $s_{k}^{\prime}$, we define a partition of $s_{k}$ by means of its sizes as

$$
s_{k}^{h} \doteq s_{k}^{\prime h} \quad \text { if } \quad h=1, \ldots, \bar{\ell},
$$

where $\bar{\ell} \doteq \max \left\{h \leq \ell^{\prime}: \sum_{q=1}^{h} s_{k}^{\prime q}=s_{k}\right\}$. Such partitions continue to satisfy the bounds (4.1), (4.3) and the one-to-one correspondence at (4.2), thanks to the estimate (3.2) and to Corollary 1, and because of the inductive assumption. Therefore, even in this case the proof will be completed once we establish the estimate (4.4) on the wave speeds. Towards this goal, observe as above that the Rankine-Hugoniot speed $\lambda_{k}$ of the outgoing shock $s_{k}$ coincides with the speeds $\lambda_{k}^{h}$ of all subwaves $\lambda_{h}^{k}$ defined according with Definition 2. On the other hand, applying Lemma 1, relying on (3.23), and thanks to the Lipschitz continuity of $(u, s) \mapsto \sigma_{k}[u](s, \cdot)$, we deduce that

$$
\begin{aligned}
\lambda_{k} \cdot\left(s_{k}^{\prime}+s_{k}^{\prime \prime}\right) & =\int_{0}^{s_{k}^{\prime}+s_{k}^{\prime \prime}} \sigma(\xi) d \xi+\mathcal{O}(1) \cdot \mathcal{J}\left(s_{k}^{\prime}, s_{k}^{\prime \prime}\right) \\
& =\int_{0}^{s_{k}^{\prime}} \sigma^{\prime}(\xi) d \xi+\int_{0}^{s_{k}^{\prime \prime}} \sigma^{\prime \prime}(\xi) d \xi+\mathcal{O}(1) \cdot \mathcal{J}\left(s_{k}^{\prime}, s_{k}^{\prime \prime}\right) \\
& =\int_{0}^{s_{k}^{\prime}+s_{k}^{\prime \prime}} \sigma^{\prime}(\xi) d \xi+\int_{0}^{s_{k}^{\prime \prime}}\left[\sigma^{\prime \prime}(\xi)-\sigma^{\prime}\left(\xi+s^{\prime}\right)\right] d \xi+\mathcal{O}(1) \cdot\left|s_{k}^{\prime} s_{k}^{\prime \prime}\right| \\
& =\int_{0}^{s_{k}^{\prime}+s_{k}^{\prime \prime}} \sigma^{\prime}(\xi) d \xi+\mathcal{O}(1) \cdot\left|s_{k}^{\prime} s_{k}^{\prime \prime}\right|,
\end{aligned}
$$


which, because of (4.9), yields

$$
\lambda_{k} \cdot\left(s_{k}^{\prime}+s_{k}^{\prime \prime}\right)=\sum_{h=1}^{\bar{\ell}} s_{k}^{\prime h} \lambda_{k}^{\prime h}+\mathcal{O}(1) \cdot\left|s_{k}^{\prime} s_{k}^{\prime \prime}\right|
$$

Hence, observing as in 1 . that by the monotonicity property of $\sigma^{\prime}(\cdot)$ we have

$$
\lambda_{k} \leq \lambda_{k}^{\prime h}+\mathcal{O}(1) \cdot \mathcal{J}\left(s_{k}^{\prime}, s_{k}^{\prime \prime}\right) \quad \forall h
$$

using (3.23), (4.18) we derive

$$
\begin{aligned}
\left|\lambda_{k}^{\prime h}-\lambda_{k}\right| & =\lambda_{k}^{\prime h}-\lambda_{k}+\mathcal{O}(1) \cdot \mathcal{J}\left(s_{k}^{\prime}, s_{k}^{\prime \prime}\right) \\
& =\frac{1}{s_{k}^{\prime}+s_{k}^{\prime \prime}} \cdot \sum_{p=1, \ldots, \bar{\ell}} s_{k}^{\prime p}\left(\lambda_{k}^{\prime h}-\lambda_{k}^{\prime p}\right)+\mathcal{O}(1) \cdot \frac{\left|s_{k}^{\prime} s_{k}^{\prime \prime}\right|}{s_{k}^{\prime}+s_{k}^{\prime \prime}},
\end{aligned}
$$

which, in turn, yields

$$
\sum_{h=1}^{\bar{\ell}} s_{k}^{\prime h}\left|\lambda_{k}^{\prime h}-\lambda_{k}\right|=\frac{1}{s_{k}^{\prime}+s_{k}^{\prime \prime}} \cdot \sum_{h=1}^{\bar{\ell}} \sum_{p=1}^{\bar{\ell}} s_{k}^{\prime h} s_{k}^{\prime p}\left(\lambda_{k}^{\prime h}-\lambda_{k}^{\prime p}\right)+\mathcal{O}(1)\left|s_{k}^{\prime} s_{k}^{\prime \prime}\right|
$$

Since the terms in the sum on the right hand-side of (4.19) are antisimmetric in $(h, p)$, the whole sum vanishes. Hence, applying (3.46), from (4.19) we recover

$$
\begin{aligned}
\sum_{h=1}^{\bar{\ell}} s_{k}^{\prime h}\left|\lambda_{k}^{\prime h}-\lambda_{k}\right| & =\mathcal{O}(1)\left|s_{k}^{\prime} s_{k}^{\prime \prime}\right| \\
& =\mathcal{O}(1) \cdot \mathcal{C}\left(s_{k}^{\prime}, s_{k}^{\prime \prime}\right) \\
& =\mathcal{O}(1) \cdot|\Delta \Upsilon(i \varepsilon)|,
\end{aligned}
$$

which proves (4.4) relying on the inductive assumption. This concludes the proof of the proposition.

\section{Conclusion}

Relying on the results established in the previous section, one can now conclude the proof of Theorem 1 following the same strategy adopted in $[5,12]$. We briefly recall it for completeness. 
Step 1. We use the partition of waves of an approximate solution $u^{\varepsilon}$ into

$$
\text { primary waves }\left\{\widetilde{y}_{k}^{h}, \widetilde{\lambda}_{k}^{h}\right\}, \quad \text { secondary waves }\left\{\widetilde{\widetilde{y}}_{k}^{h}, \widetilde{\widetilde{\lambda}}_{k}^{h}\right\},
$$

provided by Proposition 2 to construct a piecewise constant approximation

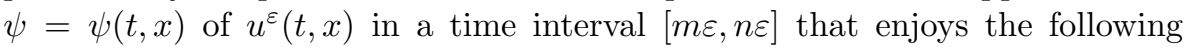
properties (cfr. [12, Section 4]).

1. The wave fronts in $\psi$ are of two kinds, primary and secondary..

2. There is a one-to-one correspondence between primary fronts and primary waves $\left\{\widetilde{y}_{k}^{h}\right\}$, and the primary front corresponding to $\widetilde{y}_{k}^{h}(m, j)$ has constant size $\widetilde{s}_{k}^{h}(m, j)$.

3. Each primary front originates at $t=m \varepsilon$ and ends at $t=n \varepsilon$. In particular, the primary front corresponding to $\widetilde{y}_{k}^{h}(m, j)$ joins the points $(m \varepsilon, j \varepsilon)$ and $\left(n \varepsilon, \ell_{(n, j, k, h)} \varepsilon\right)$ of the $(t, x)$ plane.

4. The left and right states of the primary front corresponding to $\widetilde{y}_{k}^{h}(m, j)$, say $u_{k}^{h, L}(m, j), u_{k}^{h, R}(m, j)$, are always related by

$$
u_{k}^{h, R}(m, j)=T_{k}\left[u_{k}^{h, L}(m, j)\right]\left(\widetilde{s}_{k}^{h}(m, j)\right) .
$$

Moreover, there holds

$$
\psi(m \varepsilon)=u^{\varepsilon}(m \varepsilon) .
$$

5. Let $u_{\beta}^{L}(t)$, and $u_{\beta}^{R}(t)$ be the left and right states of a secondary front $x_{\beta}(t)$ of $\psi$ at time $t \in[m \varepsilon, n \varepsilon]$. Then, letting $C W$ denote the set of all pairs of crossing primary waves in $u^{\varepsilon}$ (i.e. all pair of waves $\widetilde{y}_{k}^{h}(m, j), \widetilde{y}_{k^{\prime}}^{h^{\prime}}\left(m, j^{\prime}\right)$ for which $j<j^{\prime}, k>k^{\prime}$ and $\left.\ell_{(n, j, k, h)} \geq \ell_{\left(n, j^{\prime}, k^{\prime}, h^{\prime}\right)}\right)$, there holds

$$
\begin{aligned}
\sum_{\beta}\left|u_{\beta}^{R}(t)-u_{\beta}^{L}(t)\right| & =\mathcal{O}(1) \cdot\left[\sum_{j, k, h}\left|\widetilde{s}_{k}^{h}(m, j)\right|+\sum_{C W}\left|\widetilde{s}_{k}^{h}(m, j) \widetilde{s}_{k^{\prime}}^{h^{\prime}}\left(m, j^{\prime}\right)\right|\right] \\
& =\mathcal{O}(1) \cdot\left|\Delta \Upsilon^{m, n}\right|,
\end{aligned}
$$

where the summand on the left hand side runs over all secondary fronts in $\psi(t)$, while the second summand on the right hand side runs over all pairs of crossing primary waves in $u^{\varepsilon}$.

6. All secondary fronts travel with speed 2 , strictly larger than all characteristic speeds.

Step 2. Using the same arguments of [12, Section 5], relying on (1.12), (1.16), (4.3), (4.4), one can prove that

$$
\begin{aligned}
\left\|S_{(n-m) \varepsilon} \psi(m \varepsilon)-\psi(n \varepsilon)\right\|_{\mathbb{L}^{1}} & \\
& =\mathcal{O}(1) \cdot\left[\left|\Delta \Upsilon^{m, n}\right|+\frac{1+\log (n-m)}{n-m}+\varepsilon\right](n-m) \varepsilon \\
\left\|u^{\varepsilon}(n \varepsilon)-\psi(n \varepsilon)\right\|_{\mathbb{L}^{1}} & =\mathcal{O}(1) \cdot \Delta\left|\Upsilon^{m, n}\right| \cdot(n-m) \varepsilon
\end{aligned}
$$

where $S_{(n-m) \varepsilon} \psi(m \varepsilon)$ is the semigroup trajectory of (1.4), with initial datum $\psi(m \varepsilon)=u^{\varepsilon}(m \varepsilon)$, evaluated at time $t=(n-m) \varepsilon$. 
Step 3. As in $[12$, Section 6$]$, let $T=\bar{m} \varepsilon+\varepsilon^{\prime}$, for some $\bar{m} \in \mathbb{N}, 0 \leq \varepsilon^{\prime}<\varepsilon$, and fix a positive constant $\rho>2 \varepsilon$. Then, we inductively define integers $0=m_{0}<m_{1}<\ldots<m_{\kappa}=\bar{m}$ with the following procedure. Assuming $m_{i}$ given:

1. if $\Upsilon\left(m_{i} \varepsilon\right)-\Upsilon\left(\left(m_{i}+1\right) \varepsilon\right) \leq \rho$, let $m_{i+1}$ be the largest integer less or equal to $\bar{m}$ such that $\left(m_{i+1}-m_{i}\right) \varepsilon \leq \rho$ and $\Upsilon\left(m_{i} \varepsilon\right)-\Upsilon\left(m_{i+1} \varepsilon\right) \leq \rho$;

2. if $\Upsilon\left(m_{i} \varepsilon\right)-\Upsilon\left(\left(m_{i}+1\right) \varepsilon\right)>\rho$, set $m_{i+1} \doteq m_{i}+1$.

On every interval $\left[m_{i} \varepsilon, m_{i+1} \varepsilon\right]$ where 1 . holds, we construct a piecewise constant approximation of $u^{\varepsilon}$ according to Step 1. Then, using (5.1) we derive

$$
\begin{aligned}
& \left\|u^{\varepsilon}\left(m_{i+1} \varepsilon\right)-S_{\left(m_{i+1}-m_{i}\right) \varepsilon} u^{\varepsilon}\left(m_{i} \varepsilon\right)\right\|_{\mathbb{L}^{1}} \\
& \quad=\mathcal{O}(1) \cdot\left[\left|\Delta \Upsilon^{m_{i}, m_{i}+1}\right|+\frac{1+\log \left(m_{i+1}-m_{i}\right)}{m_{i+1}-m_{i}}+\varepsilon\right]\left(m_{i+1}-m i\right) \varepsilon .
\end{aligned}
$$

On the other hand, on each interval $\left[m_{i} \varepsilon, m_{i+1} \varepsilon\right]$ where 2 . is verified, by the Lipschitz continuity of $u^{\varepsilon}$ and applying (1.16) we find

$$
\left\|u^{\varepsilon}\left(m_{i+1} \varepsilon\right)-S_{\left(m_{i+1}-m_{i}\right) \varepsilon} u^{\varepsilon}\left(m_{i} \varepsilon\right)\right\|_{\mathbb{L}^{1}}=\mathcal{O}(1) \cdot \varepsilon .
$$

Hence, observing that the cardinality of both classes of intervals 1.-2. is bounded by $\mathcal{O}(1) \cdot \rho^{-1}$, from (5.2)-(5.3) we finally deduce

$$
\left\|u^{\varepsilon}(T)-S_{T} \bar{u}\right\|_{\mathbb{L}^{1}}=\mathcal{O}(1) \cdot\left[\rho+\frac{\varepsilon}{\rho} \log \frac{\rho}{\varepsilon}+\varepsilon\left(1+\frac{1}{\rho}\right)\right],
$$

which yields (1.15) choosing $\rho \doteq \sqrt{\varepsilon} \cdot \log |\log \varepsilon|$.

\section{References}

1. F. Ancona, A. Marson: A note on the Riemann Problem for general $n \times n$ conservation laws, J. Math. Anal. Appl. 260, 279-293 (2001).

2. F. Ancona, A. MARson: Well-posedness for general $2 \times 2$ systems of conservation laws. Memoirs Amer. Math. Soc. 169, no. 801 (2004).

3. F. Ancona, A. Marson, A wave front tracking algorithm for $N \times N$ non genuinely nonlinear conservation laws, J. Differential Equations 177, 454-493 (2001).

4. F. Ancona, A. MARson: Existence theory by front tracking for general nonlinear hyperbolic systems. Arch. Ration. Mech. Anal. 185, no. 2, 287-340 (2007)

5. F. Ancona, A. Marson: A Locally Quadratic Glimm Functional and Sharp Convergence Rate of the Glimm Scheme for Nonlinear Hyperbolic Systems. Submitted (2008).

6. S. Bianchini, On the Riemann problem for non-conservative hyperbolic systems, Arch. Ration. Mech. Anal. 166, 1-26 (2003).

7. S. Bianchini, Interaction estimates and Glimm functional for general hyperbolic systems, Discrete Contin. Dyn. Syst. 9, 133-166 (2003).

8. S. Bianchini, A. Bressan, On a Lyapunov functional relating shortening curves and viscous conservation laws, Nonlinear Analysis TMA 51(4), 649-662 (2002).

9. S. Bianchini, A. Bressan, Vanishing viscosity solutions to nonlinear hyperbolic systems, Annals of Mathematics 161, 223-342 (2005). 
10. A. Bressan, The unique limit of the Glimm scheme, Arch. Rational Mech. Anal., 130, 205-230 (1995).

11. A. Bressan, Hyperbolic Systems of Conservation Laws - The one-dimensional Cauchy problem, Oxford Univ. Press, 2000.

12. A. Bressan, A. Marson, Error bounds for a deterministic version of the Glimm scheme, Arch. Rational Mech. Anal., 142, 155-176 (1998).

13. A. BREssan, T. YANG, On the convergence rate of vanishing viscosity approximations, Comm. Pure Appl. Math, 57, 1075-1109 (2004).

14. C.M. Dafermos, Hyperbolic conservation laws in continuum physics SpringerVerlag, Berlin, 2000.

15. O. Glass, P.G. LeFloch: Nonlinear hyperbolic systems: nondegenerate flux, inner speed variation, and graph solutions, Arch. Ration. Mech. Anal. 185, no. 3, 409-480 (2007).

16. J. GLImM, Solutions in the large for nonlinear hyperbolic systems of equations, Comm. Pure Appl. Math., 18, 697-715 (1965).

17. J. HuA, Z. JiAng AND T. YANG, A new Glimm functional and convergence rate of Glimm scheme for general systems of hyperbolic conservation laws, Conservation Laws Preprint Server, preprint 2008-015 (2008).

18. J. HuA, T. YANG, An improved convergence rate of Glimm scheme for general systems of hyperbolic conservation laws, J. Differential Equations, 231, 92-107 (2006).

19. T. IGUChi, P.G. LeFloch, Existence theory for hyperbolic systems of conservation laws with general flux-functions. Arch. Rational Mech. Anal., 168, 165-244 (2003).

20. P.D. LAx, Hyperbolic systems of conservation laws II, Comm. on Pure and Applied Math., 10, 537-566 (1957).

21. T.P. Liu, The determnistic version of the Glimm scheme, Comm. Math. Phys. 57, 135-148 (1975)

22. T.P. Liu, The Riemann problem for general $2 \times 2$ conservation laws, Trans. Amer. Math. Soc., 199, 89-112 (1974)

23. T.P. Liu, The Riemann problem for general systems of conservation laws, $J$. Differential Equations, 18, 218-234 (1975)

24. T.P. LiU, Admissible solutions of hyperbolic conservation laws, Memoirs Amer. Math. Soc., 30, no. 240 (1981).

25. T.P. LiU, T. YANG, Weak solutions of general systems of hyperbolic conservation laws, Comm. Math. Phys. 230, 289-327 (2002)

26. T. YANG, Convergence rate of Glimm scheme for general systems of hyperbolic conservation laws, Taiwanese J. Math., 7, 195-205 (2003).

Dipartimento di Matematica Pura ed Applicata

Via Trieste 63

35121 - Padova, Italy

email: ancona@math.unipd.it

and

Dipartimento di Matematica Pura ed Applicata

Via Trieste 63

35121 - Padova, Italy

email: marson@math.unipd.it 